\title{
FINITE ELEMENT MODELING OF ICD LEAD SILICONE SOFT-TIPS
}

\author{
A Thesis \\ presented to \\ the Faculty of California Polytechnic State University, \\ San Luis Obispo
}

In Partial Fulfillment

of the Requirements for the Degree

Master of Science in Engineering, with Specialization in Biomedical Engineering

by

Jose Jesus Lepe

May 2010 
(C) 2010

Jose Jesus Lepe

ALL RIGHTS RESERVED 
TITLE:

Finite Element Modeling of ICD

Lead Silicone Soft-Tips

AUTHOR: $\quad$ Jose Jesus Lepe

DATE SUBMITTED: $\quad$ May 2010

COMMITTEE CHAIR: $\quad$ Dr. Lanny Griffin, Department Chair, BMED

COMMITTEE MEMBER: $\quad$ Dr. Daniel Walsh, Senior Associate Dean, Engineering

COMMITTEE MEMBER: Dr. Scott Hazelwood, Professor of Engineering

COMMITTEE MEMBER: $\quad$ Dr. Shawn Chen, Principal Engineer,

St. Jude Medical 


\author{
ABSTRACT \\ Finite Element Modeling of ICD Lead \\ Silicone Soft-Tips \\ Jose Jesus Lepe
}

\begin{abstract}
Although highly underutilized by the medical device industry, Finite Element Analysis (FEA) in the development of new technologies is gaining popularity as regulatory bodies such as the Food and Drug Administration (FDA) begin to require additional proof of safety through scientific methods. Non-linear FEA allows engineers to realistically simulate the mechanical behavior of implants as seen in the in-vitro, or in some cases, the in-vivo configurations. The work presented in this report investigates how computational methods can be used to simulate the interaction of a St. Jude Medical silicone soft-tip as it passes through a Peel-Away Sheath (i.e. introducer). In this analysis the soft-tips were modeled as axisymmetric with hyperelastic material properties assigned to the soft-tips. An Ogden, second order hyperelastic material model was used to describe the non-linear stress-strain behavior of silicone soft-tips. The finite element program, ABAQUS/Standard was used to simulate the soft-tip/introducer interactions. The reaction forces obtained through these simulations represent the force required to push a lead through an introducer, and were then compared to experimental data.
\end{abstract}

Keywords: Finite Element Analysis, FEA, Hyperelastic, Abaqus, Silicone, Implantable Cardioverter Device, ICD, Soft Tip. 


\section{ACKNOWLEDGEMENTS}

I would like to extend my sincerest gratitude to all those who have helped me achieve this great milestone in my life. My deepest appreciation to my committee chair Dr. Lanny Griffin, who has provided me with valuable insight and guidance in this endeavor from the beginning of my studies at Cal Poly. To Dr. Shawn Chen, Dr. Daniel Walsh, and Dr. Scott Hazelwood, my thanks for their support that has led me to the successful completion of my research.

I am very grateful to Phong Doan and Dr. Yong Zhao, who have guided me in my first years as an engineer at St. Jude Medical. I am also grateful to my colleagues at St. Jude Medical who have all contributed to my success over the years. Being at St. Jude Medical has been a wonderful experience and is only made possible by those who take pride in their work.

I would also like to thank the engineering staff at Abaqus and NuSil Silicone Technologies for providing me with the advice and information necessary to complete my research.

I finish with my family, whose support has been unconditional all these years. My parents have made many sacrifices for me to be where I am today, and I cherish them for that. They have been the greatest teachers in my life, and continue to be my inspiration. Finally and foremost, I would like to thank my dear wife, Marisol. From the moment I applied to graduate school her support and encouragement have never failed. Thank you for always being by my side. 
LIST OF TABLES

LIST OF FIGURES

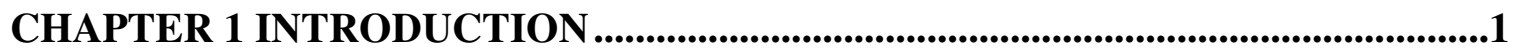

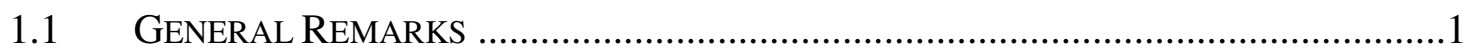

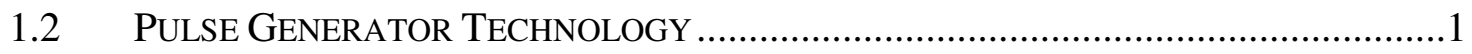

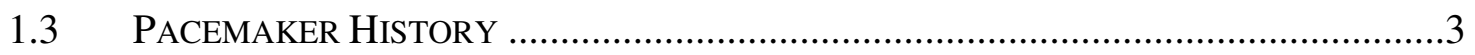

1.4 ICD HISTORY

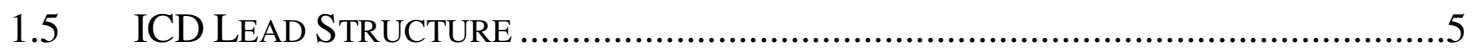

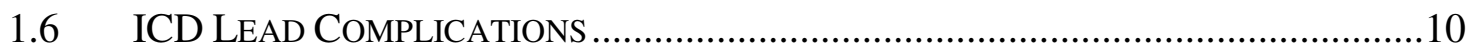

1.6.1 Lead Dislodgement ....................................................................................11

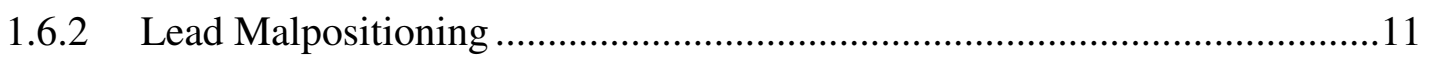

1.6.3 Lead Perforation...................................................................................12

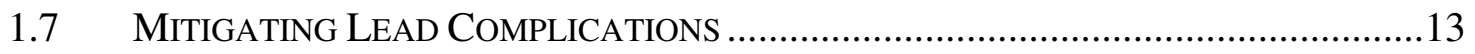

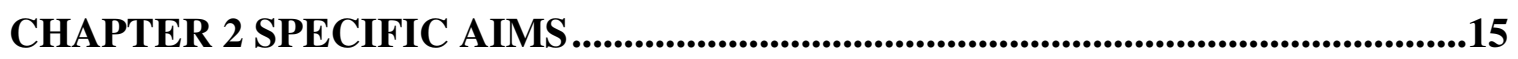

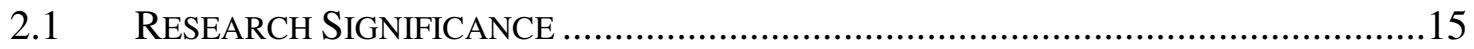

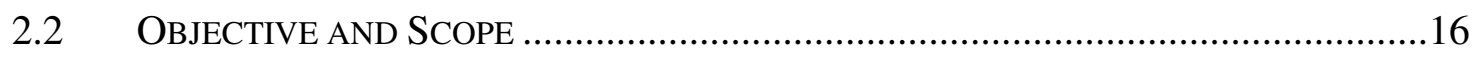

CHAPTER 3 METHODS AND MATERIALS.......................................................................17

3.1 GENERAL REMARKS ……………………........................................17

3.2 Comparison BeTweEn Physical AND FEA Soft-TIPS.....................................18

3.2.1 Basic Assumptions .....................................................................................18 
3.2.2 Hyperelastic Material Models .............................................................20

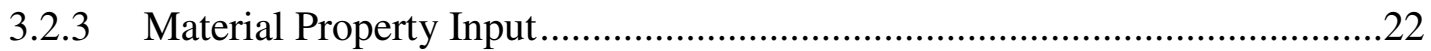

3.3 MODELING OF THE 7F DuRATA ICD LEAD SOFT-TIP .....................................25

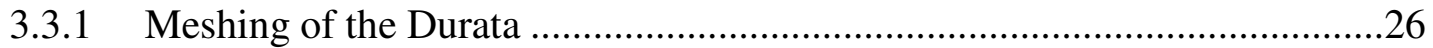

3.3.2 Loading and Boundary Conditions of the Durata and 7F Introducer ...........27

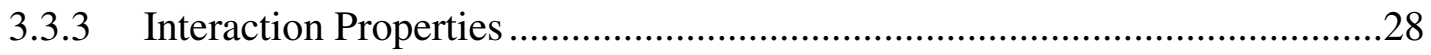

3.4 MOdELING OF THE 6F ADVANCED ICD LEAD SOFT-TIP ...................................31

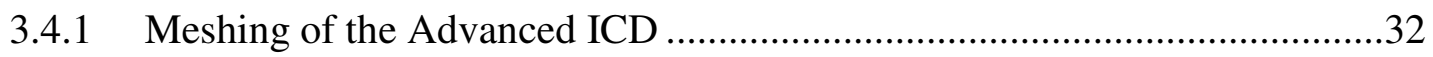

3.4.2 Loading and Boundary Conditions of the Advanced ICD and 6F

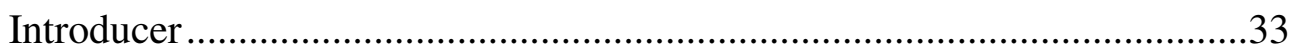

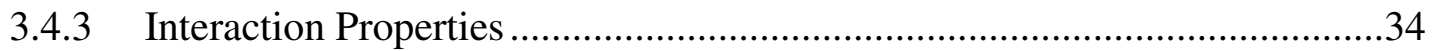

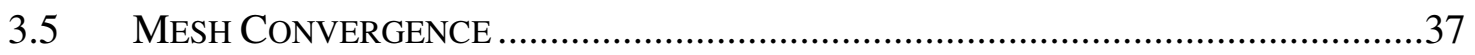

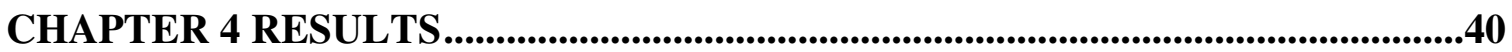

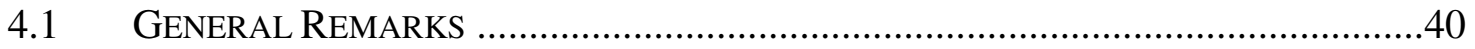

4.2 ANALYSIS RESUltS OF THE DURATA SOFT-TIP ..........................................40

4.3 ANALYSIS RESUltS OF THE ADVANCED ICD SOFT-TIP ..................................43

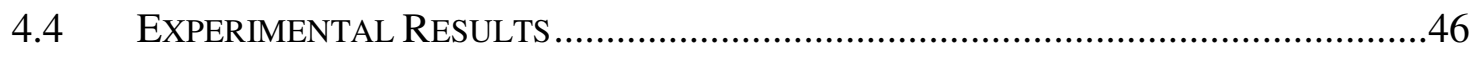

4.5 THEORETICAL AND EXPERIMENTAL COMPARISONS ....................................48

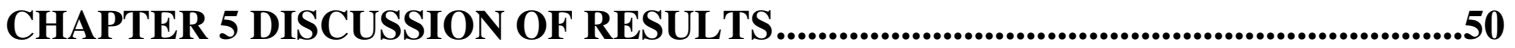

CHAPTER 6 FUTURE DIRECTIONS AND CONCLUSIONS ................................52

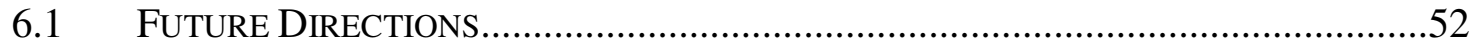

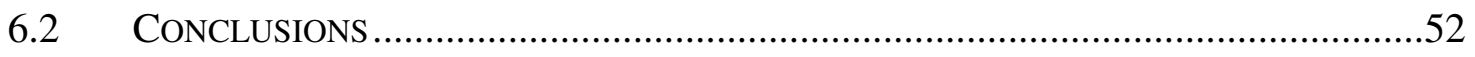




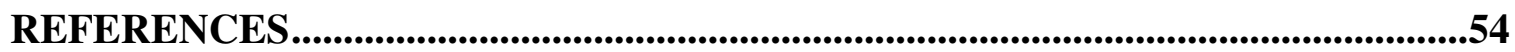

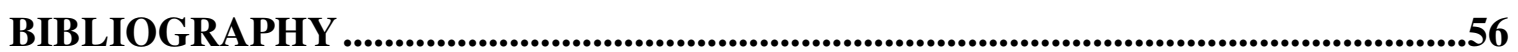




\section{LIST OF TABLES}

Table

Table 3-1. Physical properties of Med-4860.

Table 3-2. Interaction properties between the Soft-Tip and Introducer.

Table 3-3. Interaction properties between the Soft-Tip and Introducer.

Table 3-4. Summary of mesh refinement for Durata Soft-Tip model.

Table 3-5. Summary of mesh refinement for Advanced ICD Soft-Tip model..................37

Table 4-1. Experimental lead introducer test for 7F Durata ICD lead............................47

Table 4-2. Experimental lead introducer test for 6F Advanced ICD lead. ........................48 


\section{LIST OF FIGURES}

Figure Page

Figure 1-1. The conduction system of the heart. ...................................................

Figure 1-2. SJM Integrity ADx XL DR pacemaker and Atlas II DR ICD. ......................

Figure 1-3. General ICD lead placement in the heart chambers..................................6

Figure 1-4. SJM Riata ST defibrillation lead.........................................................

Figure 1-5. Cross-Section of SJM Riata ST lead body...........................................

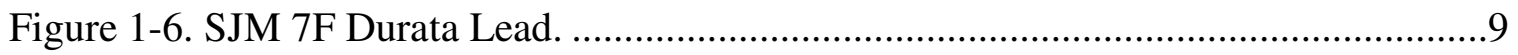

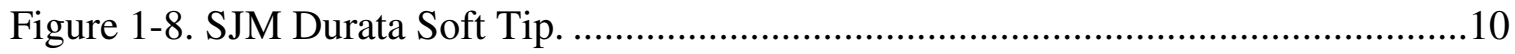

Figure 1-9. Perforation of the posterior leaflet of the mitral valve [14]. .........................12

Figure 3-1. Modeling and simulation methodology. ...............................................18

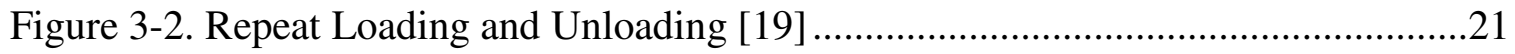

Figure 3-3. A tension experiment using a video extensometer...................................23

Figure 3-4. State of stress of uniaxial tensile test. ...................................................23

Figure 3-5. Automatic material evaluation procedure in Abaqus.................................24

Figure 3-6. Stress-Strain plots for uniaxial response ............................................25

Figure 3-7. Geometry of the SJM 7F Durata ICD Soft-Tip........................................26

Figure 3-8. Simplified 7-French Peel-Away Sheath...............................................26

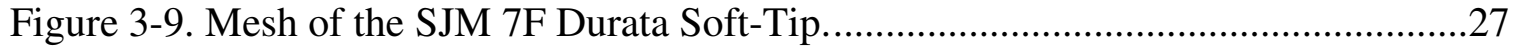

Figure 3-10. Boundary conditions at soft-tip/marker band interface of Durata model. ....28

Figure 3-11. Exponential decay friction model specified with test data points................29

Figure 3-12. Surface constraints of Introducer and Durata Soft-Tip..............................30

Figure 3-13. Self-Contact surface of the SJM Durata soft-tip.......................................31 
Figure 3-14. Geometry of the 6F Advanced ICD Soft-Tip..........................................

Figure 3-15. Simplified 6-French Peel-Away Sheath................................................32

Figure 3-16. Mesh of the 6F Advaced ICD Soft-Tip.................................................33

Figure 3-17. Boundary conditions of Advanced ICD model at soft-tip/marker

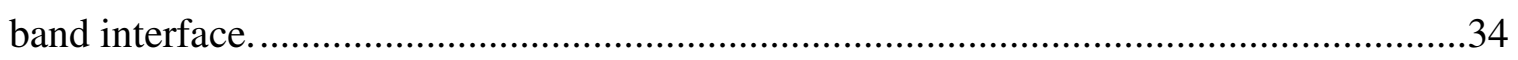

Figure 3-18. Surface constraints of Introducer and Advanced ICD Soft-Tip...................36

Figure 3-19. Self-Contact surface of Advanced ICD Soft-Tip....................................36

Figure 3-20. Mesh convergence curve for Durata Soft-Tip model..................................38

Figure 3-21. Mesh convergence curve for Advanced ICD Soft-Tip model. ....................39

Figure 4-1. Durata Mises stress distribution across the soft-tip at $\mathrm{t}=0.719 \mathrm{sec} . \ldots \ldots \ldots \ldots . . .41$

Figure 4-2. Displacement of the Durata soft-tip during insertion..................................41

Figure 4-3. Durata Mises stress distributions for 0.0008 and 0.0020 seeding ratios........42

Figure 4-4. Simulated push force of the 7F Durata Soft-Tip and 7F Introducer models...43

Figure 4-5. Advanced ICD Mises stress distribution at steps 0, 20, 40, and 60.............44

Figure 4-6. Displacement of the Advanced ICD soft-tip during insertion. .....................45

Figure 4-7. Simulated push force of the 6F Advanced ICD Soft-Tip and 6F

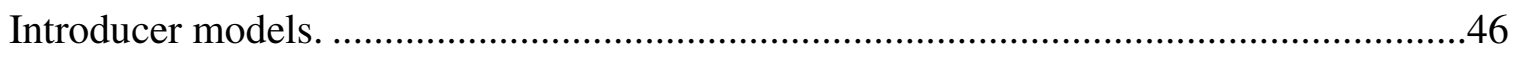

Figure 4-8. FEA and experimental maximum push force for Durata Soft-Tip. ...............49

Figure 4-9. FEA and experimental maximum push force for Advanced ICD Soft-Tip...49 


\section{CHAPTER 1 INTRODUCTION}

\subsection{GENERAL REMARKS}

Since 1958 implantable Pacemakers, and later on Implantable Cardioverter Defibrillators (ICD's), have been used to detect arrhythmias and help the heart beat in a regular rhythm. The insulated cardiac electrodes, also known as leads, are tasked with relaying information from the heart to the device and delivering electrical therapy pulses to the heart wall as needed. The leads must be small and flexible enough to pass through the veins, yet durable enough to withstand the harsh environment for a number of years. A stiff lead can result in the distal tip perforating the heart wall, while a poorly insulated lead can result in complications such as corrosion, fracture, and loss of sensing. Optimizing for flexibility and durability can be a difficult task, but necessary to ensure the safety of the patient. The silicone soft-tip is a feature implemented in St. Jude Medical (SJM) ICD leads to provide a cushion against the heart wall, minimizing the tip stiffness of an ICD lead. By optimizing the surface area and thickness of the soft-tip we inherently reduce tip stiffness and therefore reduce the risk of cardiac perforation.

\subsection{PULSE GENERATOR TECHNOLOGY}

As the "pump" of the cardiovascular system the heart is regulated by a conduction system that activates the chambers of the heart in a synchronous rhythm. The sinoatrial (SA) node, located on the high right atrium of the heart (Figure 1-1), has specialized cells with the ability to generate electricity [1]. Considered the heart's natural pacemaker, the 
SA node delivers electrical pulses down the conduction path to the atrioventricular (AV) node, which acts as a relay station, delaying the pulse then delivering it to the ventricles.

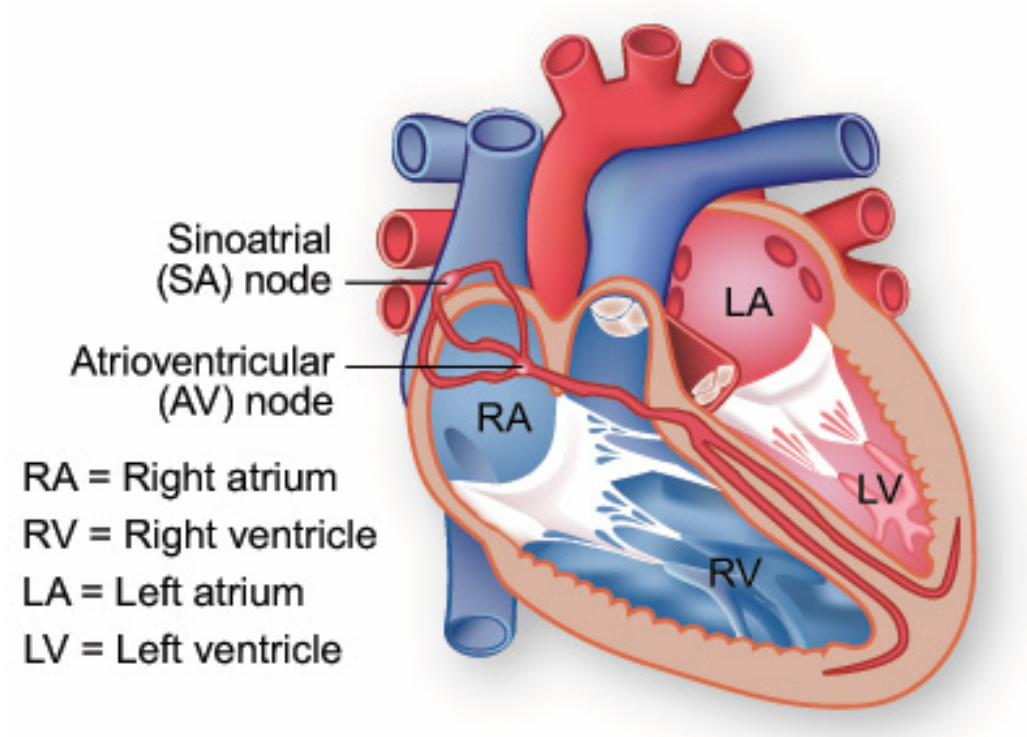

Figure 1-1. The conduction system of the heart.

At times the SA node may be defective, causing the heart to beat too fast, too slow, or in an irregular pattern. The artificial pacemaker serves to correct conduction disorders and treats arrhythmias such as bradycardia by sending electrical therapy-pacing pulses to help the heart beat in a regular rhythm. Similar to a pacemaker in that many can also treat bradycardia, ICD's primarily treat tachyarrhythmias by delivering defibrillation therapy as necessary. Shown in Figure 1-2 are a St. Jude Medical Integrity Pacemaker and Atlas II ICD [2]. 

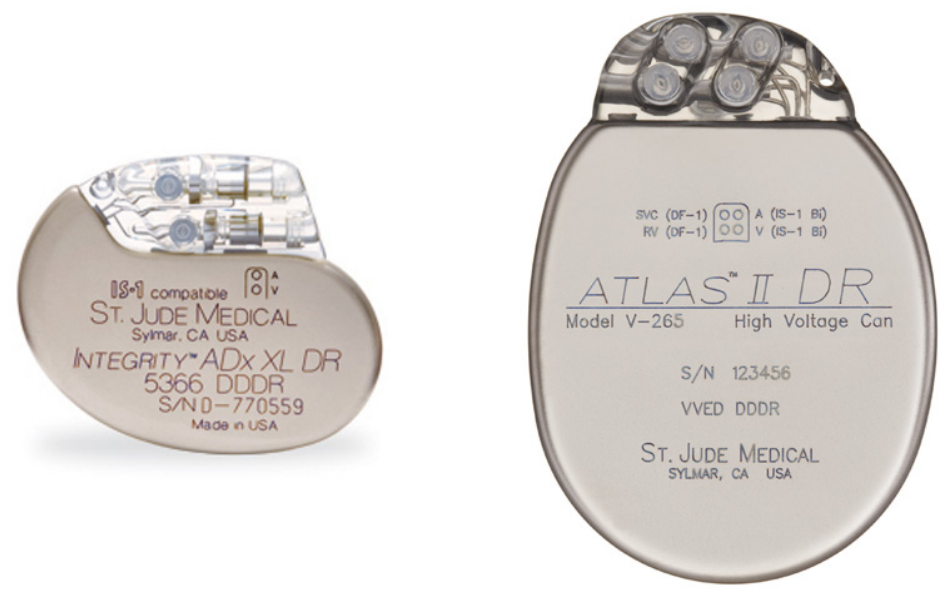

Figure 1-2. SJM Integrity ADx XL DR pacemaker and Atlas II DR ICD.

\subsection{PACEMAKER HISTORY}

Stimulation of muscle tissue by electrical means was documented as early as the mid- $18^{\text {th }}$ century. In 1771 , Luigi Galvani, an Italian scientist and physician, discovered that the muscles of dead frogs twitched when electrically stimulated. Galvani later discovered that muscles, including the heart, stopped responding to electrical stimulation shortly after being deprived of blood [3].

Throughout the $19^{\text {th }}$ century much research was devoted to the research of human physiology. In 1804, Giovanni Aldini published a highly influential book incorporating the principles of Luigi Galvani (animal electricity) and Alessandro Volta (bimetallic electricity) into Aldini's experiments. Aldini's attempts to demonstrate the involvement of the electrical fluid in muscle contraction in frogs eventually led to an essay entitled: Commentary on the Effects of Electricity on Muscular Motion. Aldini used Volta's bimetallic pile to electrically stimulate the hearts of recently executed people [4]. 
Guillaume-Benjamin-Amand Duchenne de Boulogne, was a French neurologist who revived Galvani's research and pioneered the science of muscular electrophysiology. In 1869, Duchenne succeeded in slowing the heart rate of a patient who suffered from tachyarrhythmia [3].

Credited for coining the term "artificial pacemaker", Albert S. Hyman is widely regarded as the father of artificial pacing. In 1930, along with his brother, an engineer, Hyman developed the first artificial pacemaker, an electro-mechanical instrument powered by a spring-wound hand-cranked motor. Electrical impulses supplied to the heart via a bipolar needle introduced through the chest wall [5], [3].

On August 28, 1952 Dr. Paul M. Zoll performed the first human clinical cardiac pacing in Boston, Massachusetts. The Zoll pacemaker was an external pacemaker designed to stimulate across the closed chest. The two electrodes were metal disks strapped onto the chest. Treatment with the Zoll pacemaker was only for emergency purposes because the stimulation was painful and required sedation [5], [3].

The first implantable pacemaker was invented by a Swedish engineer named Rune Emqvist and was implanted in Arne Larson on October 8, 1958. Designed to treat Stokes-Adams Syndrome, the first pacemaker implanted in Mr. Larson lasted only three hours, and was replaced the next day with a second device which lasted six weeks. These devices would be only two of the 22 devices Mr. Larson received over his lifetime. 
Initially about the size of a hockey puck, the pacemaker continues to evolve by becoming smaller and more advanced over the years [6].

\subsection{ICD HISTORY}

It is believed that Sudden Cardiac Death (SCD) is responsible for nearly one fourth of all human deaths [7], and was the cause of death of Dr. Michel Mirowski's dear friend. Dr. Mirowski is credited as the inventor of the ICD, and has endured much criticism from the medical community during his initial efforts to develop the ICD.

In 1969, only three years after the death of Dr. Mirowski's friend and colleague, Mirowski began his research on the ICD with Dr. Morton Mower [6]. By 1970 Dr. Mirowski submitted and was granted a US Patent the concept of a totally implanted defibrillator system. The system used intracardiac catheter and SQ patch, and detection via RV pressure transducer. After years of animal research and extensive investigational review board inquiries, Dr. Mirowski and his team received permission for implants. In 1980, the first ICD was successfully implanted at John Hopkins Hospital by Dr. Levi Watkins. By 1985 the FDA cleared the ICD for commercial sales in the US [6].

\subsection{ICD LEAD STRUCTURE}

The modern ICD system consists of three main components; the ICD device (pulse generator), the leads (electrodes), and the programmer shown in Figure 1-3 [2]. 


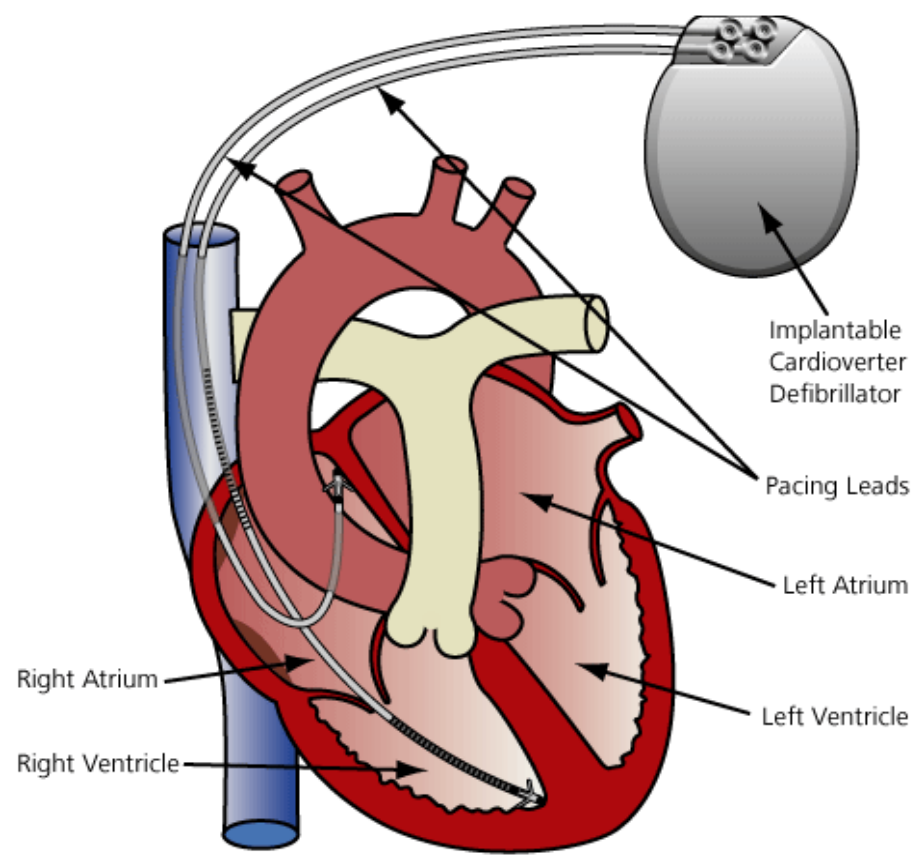

Figure 1-3. General ICD lead placement in the heart chambers.

As the ICD device generates the pacing pulses or defibrillation shocks, the required therapy is transmitted to the heart tissue via the leads. While the design of transvenous leads vary among manufacturers, with the exception of standardized connectors in defibrillation leads, their basic construction consists of 1) the electrode(s), 2) the conductor(s), 3) insulation, 4) the connector(s), and 5) the fixation mechanism [8]. Shown in Figure 1-4 is the St. Jude Medical Riata ST defibrillation lead [2]. 


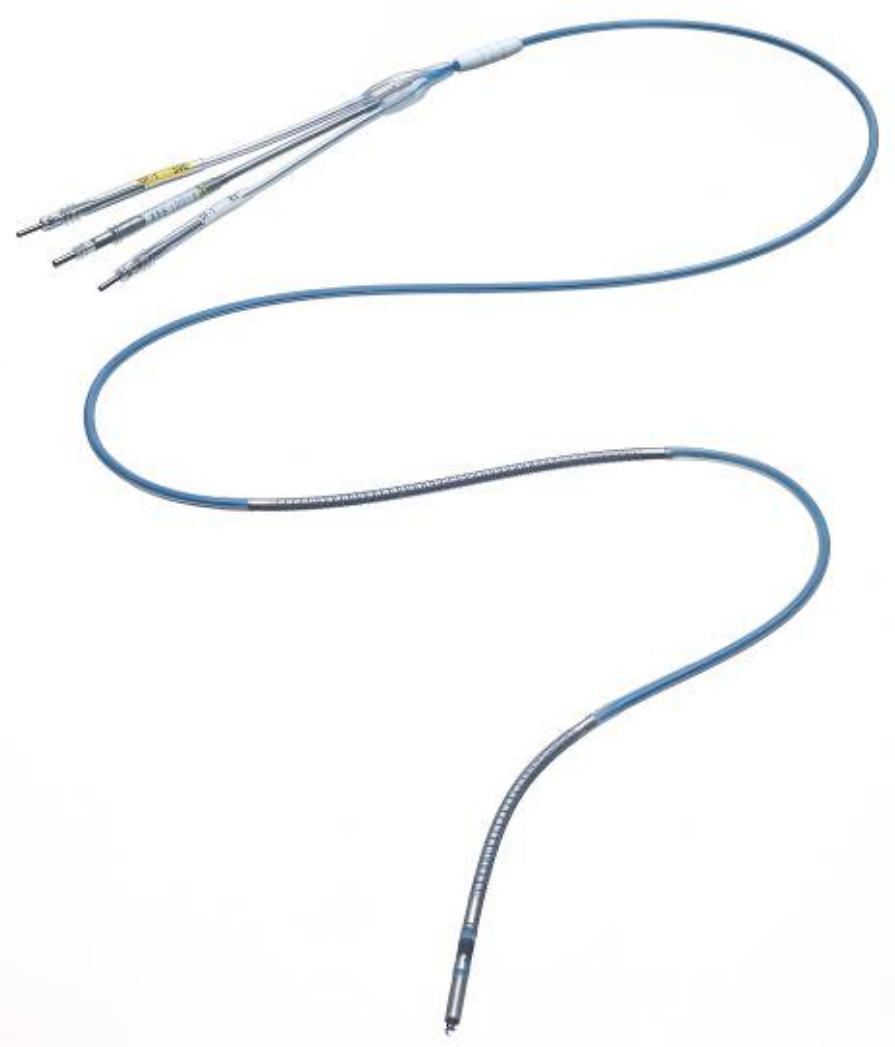

Figure 1-4. SJM Riata ST defibrillation lead.

St. Jude Medical defibrillation leads utilize a concentric lead body design, where the lumens in the insulation are concentric about a single center lumen (Figure 1-5). In general, defibrillation leads are larger in diameter than pacing leads due to thicker insulation and conductors. The larger conductors and insulators allow the lead to deliver high-energy shocks without compromising the integrity of the lead. 


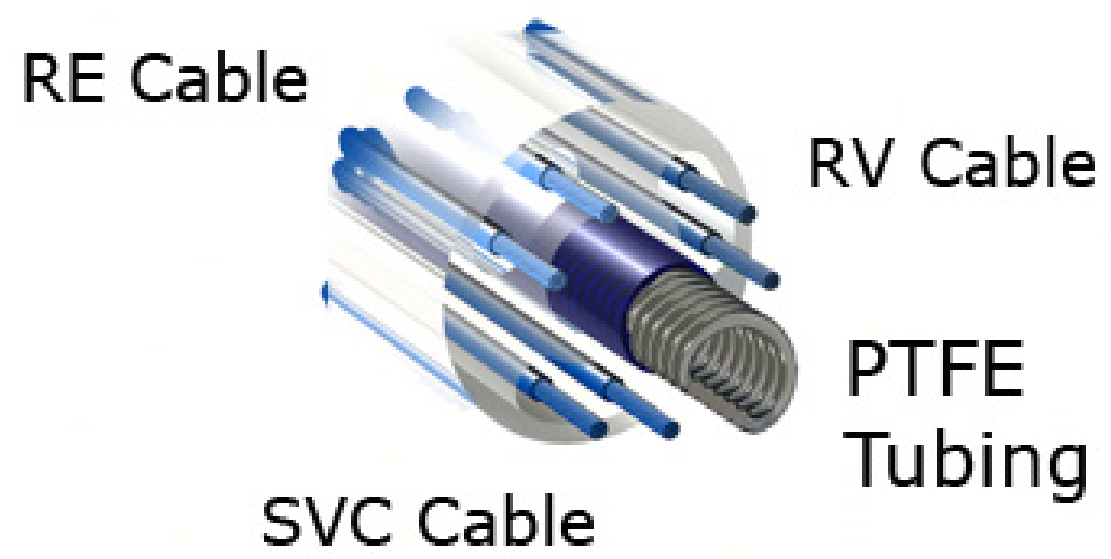

Figure 1-5. Cross-Section of SJM Riata ST lead body.

Figure 1-6 shows a close-up of the distal end of a St. Jude Medical, 7F Durata ICD lead along with other key features of this lead [2]. The exposed shocking coils deliver the defibrillation therapy to the heart chambers. An inner coil connects a connector pin to the helix and allows torque to be transferred through the lead body; enabling the helix to be extended into the heart wall by the surgeon. The soft-tip, which is the primary focus of this project, greatly reduces tip pressure as the distal tip of the lead presses against the heart wall. 


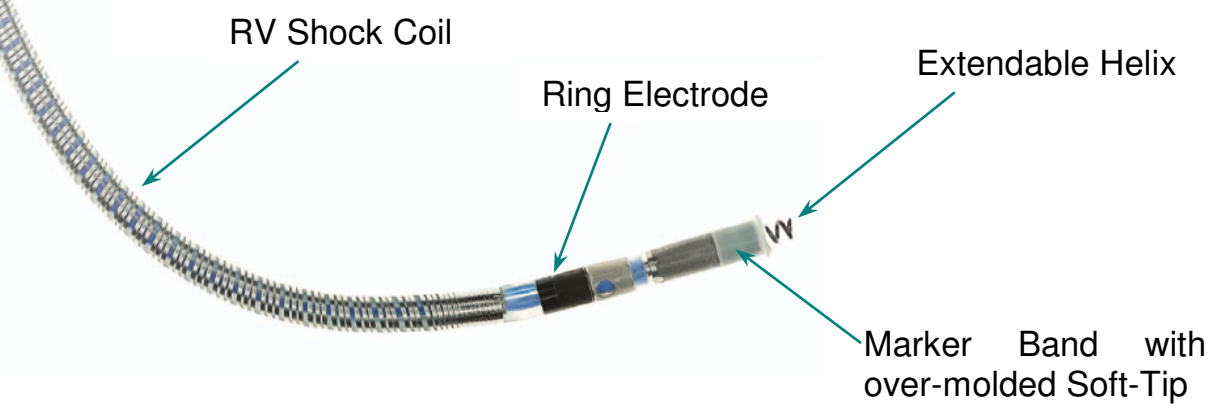

Figure 1-6. SJM 7F Durata Lead.

Stable positioning of the lead is critical to its long-term performance. To keep the distal tip of the lead from becoming dislodged it must be anchored to the heart wall. Although several mechanisms have been designed to aid in fixation of the lead, there are two fundamental endocardial fixation methods - passive and active fixation (Figure 1-7).

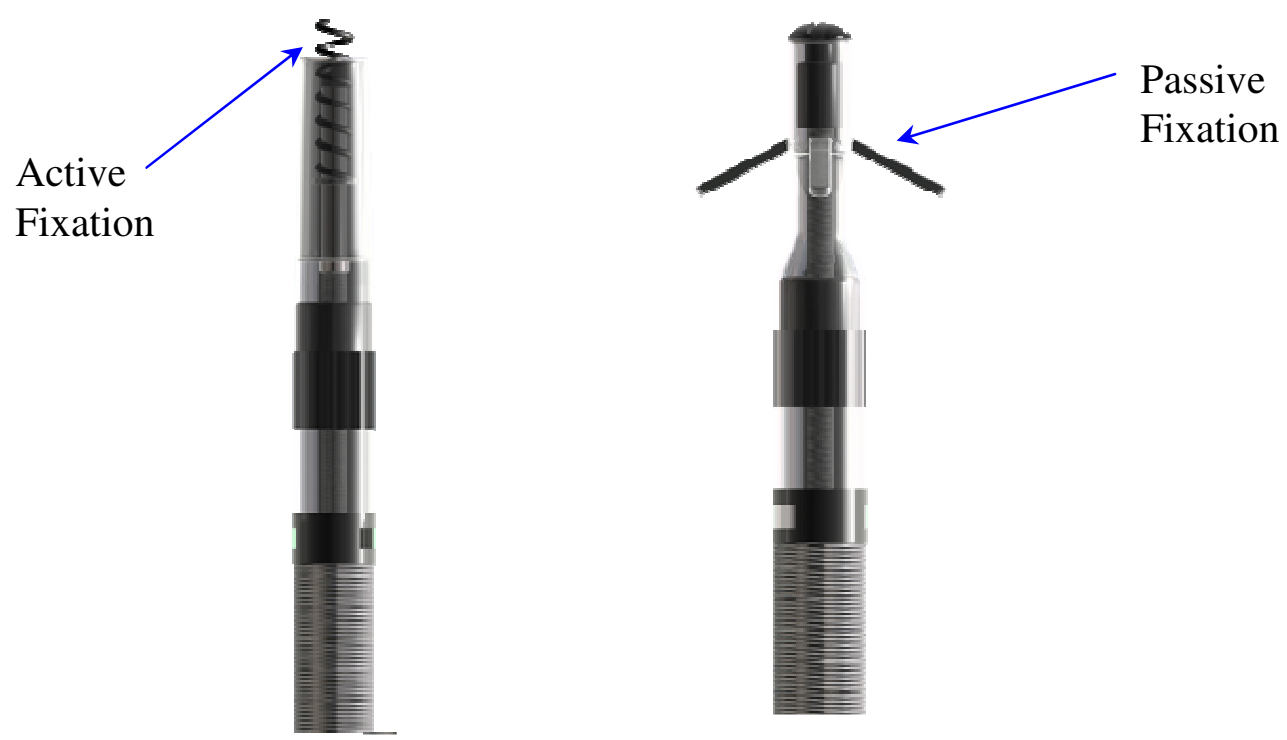

Figure 1-7. SJM 7F Riata ST leads with active and passive fixation tips. 
Passive fixation lead tips are extensions of the insulation and are designed to become lodged within the trabeculae of the right atrium or ventrical. The primary active fixation method used today is the screw-in helix that is extended into the endocardium.

Figure 1-8 shows the distal tip of a 7F Durata with a retracted helix. The soft-tip enables better conformity of the lead tip to the heart wall and reduces tip pressure. The flanged silicone tip increases the surface area to an $8 \mathrm{~F}$ diameter, but can still be collapsed and passed through a 7F introducer.

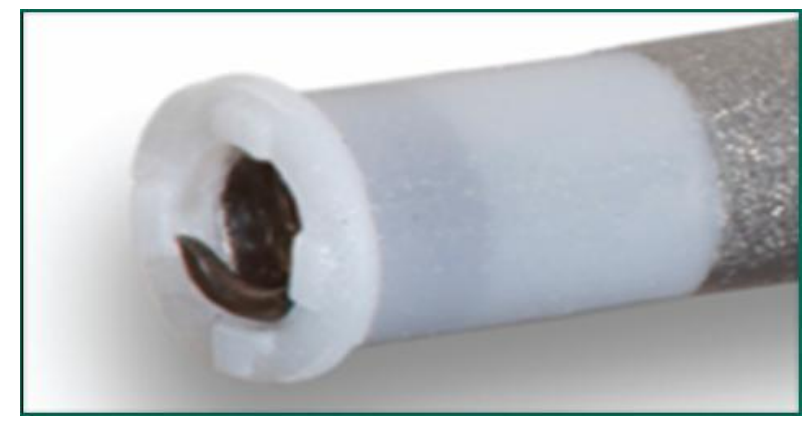

Figure 1-8. SJM Durata Soft Tip.

\subsection{ICD LEAD COMPLICATIONS}

The mortality benefits, the evolution of ICD technology, and the relative ease of implantation have all fueled the increase in ICD implantation in recent years. The evolution of ICD technology has resulted in much smaller lead diameters, with the largest lead in the industry being less than 9 French $(3 \mathrm{~mm})$. Small defibrillation leads allow for multiple lead implantations that are required for multi-site pacing, and reduces the risk of subclavian crush syndrome. Despite the benefits and relative ease of ICD implantation, lead-related complications have been reported in up to $6.9 \%$ of patients [9] and may be 
due to implant procedure or lead functionality which can cause lead dislodgement, malposition, or perforation.

\subsubsection{Lead Dislodgement}

Lead dislodgement is most common in transvenous leads, but has also been observed in other lead types such as epicardial and subcutaneous leads. Acute lead dislodgement occurs within the first six weeks of implantation [10] and may be attributed to the extension or retraction of the torso, causing the distal tip of the lead to be pulled loose from the cardiac tissue before fibrosis has fully encapsulated and secured the lead tip to the heart wall. The mechanisms responsible for late lead dislodgement are consequently "Twiddler's Syndrome", "Reel's Syndrome", or simply trauma to the pacemaker system, and usually evolve from manipulation of the device and/or lead [10]. Twiddler's Syndrome refers to the permanent malfunction of the device and is directly caused when the patient, unintentionally or deliberately, rotates the device on its long axis while in the subcutaneous pocket. Reel's Syndrome is similar to Twiddler's Syndrome, but the axis of rotation is such that the lead wraps around the device cause the distal end of the lead to become dislodged.

\subsubsection{Lead Malpositioning}

Lead malpositioning can occur in patients with cardiac structural abnormalities and may lead to several complications such as systemic thromboembolic complications or damage to normal cardiac structure by means of mitral valve or left ventricular wall perforation [11]. Cases have been reported where the pacemaker lead is inadvertently placed in the left ventricle [10-12]. Shown in Figure 1-9 is a result of a pacemaker lead malpositioning to the left ventricle through a Patent Foramen Ovale (PFO) - one type of 
interatrial septum. Other means of malpositioning through a sinus venosus atrial septal defect (ASD) or interventricular septum have also been reported [14] but are not as common as the aforementioned pathway.

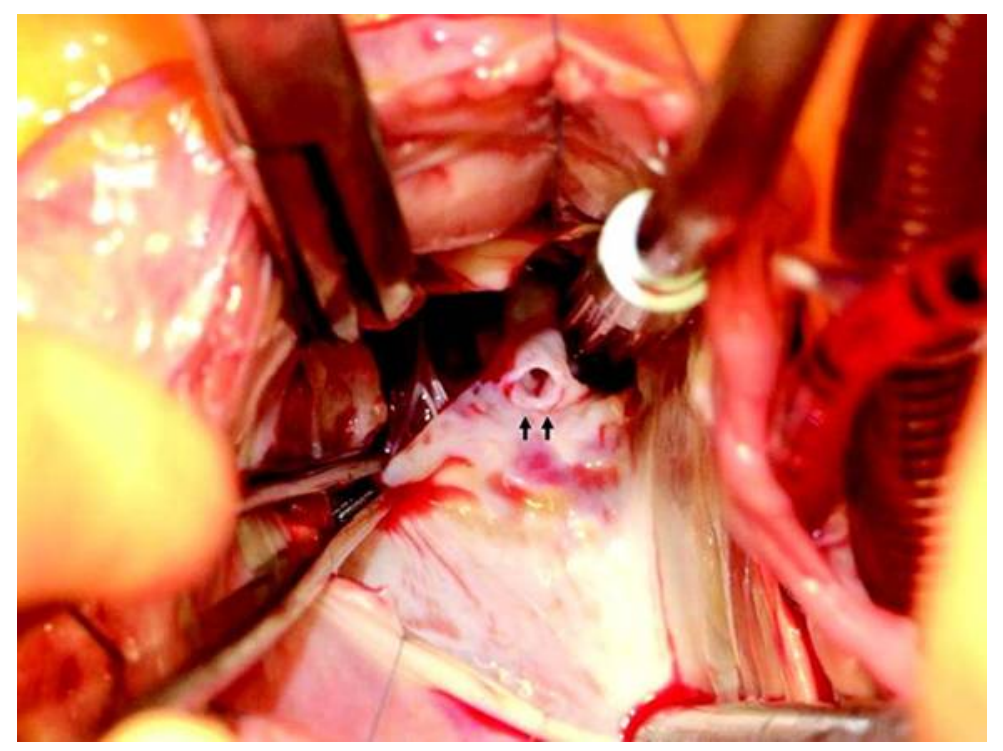

Figure 1-9. Perforation of the posterior leaflet of the mitral valve [14].

\subsubsection{Lead Perforation}

Possibly the most serious lead-related complication is perforation, a phenomenon where the lead has extended through the entire heart wall. Incidences of lead perforation are relatively low, reported to be between $0.1-0.8 \%$ for pacing leads and $0.6-5.2 \%$ for ICD leads [15], [16], but may result in severe clinical consequences including pericardial effusion, cardiac tamponade, pneumothorax and death [17]. Perforation that has occurred during implant is referred to as acute perforation, and if it is not evident at the time of implant but symptoms are found after 24 hours, the term subacute perforation applies. Perforation complications that arise more than one month post-implant are referred to as 
delayed perforation. Some cases of delayed perforation have been reported up to 10 months post-implant [18]. Lead perforation is often attributed to one or a combination of factors. Patient characteristics such as heart wall thickness, patient age, and Body Mass Index less than 20, are a few of the factors that may increase the risk of perforation. Procedural risk factors include lead/stylet stiffness, use of a temporary pacemaker, extendable helix lead fixation, lead implant techniques (including overtorque of the helix), and the design characteristics of the lead.

\subsection{MITIGATING LEAD COMPLICATIONS}

Because lead perforation can have fatal consequences many resources at St. Jude Medical have been put into mitigating this risk. As stated in the previous section, the design characteristics of the lead can have a significant impact on the risk of perforation and other lead complications. The following design characteristics have been employed to aid in reducing the risk of perforation:

- Flexible Leads (Smaller-Diameter)

- Passive vs. Active-Fixation Leads

- Soft-Tip to reduce tip stiffness

In general ICD leads are thicker and stiffer than pacing leads due their design characteristics. These high voltage leads require thicker insulation and conductors to successfully transfer the pacing/defibrillation therapy from the device to the heart. Downsizing the lead diameter of ICD leads is desirable because it can reduce the overall stiffness of the lead. The drawback to downsizing an ICD lead is that it can sacrifice lead 
durability and the ability of the conductors to carry a high current through downsized cables.

Active fixation leads utilize a screw-in helix that allows the electrode to be screwed into the heart wall. The screw-in helix is advantageous because it gives the surgeon the freedom to secure the lead to the location in the heart that will provide the most effective therapy. The disadvantage to using a screw-in helix to fixate the lead is that these tips are usually stiffer than a passive fixation lead and can increase the risk of perforation.

In order to mitigate the risk of cardiac perforation in an active fixation lead, St. Jude Medical utilizes a silicone molded soft-tip at the distal end of the lead that helps reduce the tip stiffness of an ICD active fixation lead. The soft tip is designed to offer increased surface area at the distal tip and still pass through an introducer with a maximum push force of less than two pounds. This report focuses on the soft-tip design as a risk mitigator, and the use of finite element analysis as a tool to aid in the design and optimization of the soft-tip. 


\section{CHAPTER 2 SPECIFIC AIMS}

\subsection{RESEARCH SIGNIFICANCE}

Encouraged by the Food and Drug Administration (FDA), and other regulatory bodies, modeling and simulation is increasing in popularity amongst medical device manufacturers. Given the appropriate inputs FEA can simulate the interaction between a soft-tip and an introducer, and predict stress concentrations and reaction forces. Prior to FEA, it has only been possible for the design engineer to estimate the force required to push a lead through an introducer by molding a new soft-tip and performing an experimental introducer test. Although experimental testing is preferred, it is a costly optimization method significantly increases the development time. By using FEA the design engineer can predict the performances of the part prior to making prototypes, therefore reducing the product development cycle and the costs associated with experimentation and fabrication. FEA is not intended to replace experimental testing, but intended to be used as a predictive design tool.

With regards to FEA of implantable cardiac leads, literature search is limited to the analysis of lead coils [21-22]. The silicone soft-tips are unique to St. Jude Medical active fixation leads, and as expected, a literature search by the author has revealed no information about the FEA of ICD silicone soft-tips. 


\subsection{OBJECTIVE AND SCOPE}

The aim of this master's thesis is to construct finite element models of St. Jude Medical's 7-French Durata and 6-French Advanced ICD soft tips and perform non-linear static analysis using the hyperelastic models available in the finite element software, ABAQUS v6.8-1. Through this effort the author intends to show that a finite element simulation can be used to predict, with reasonable accuracy, the force required to push a lead through a Peel-Away Sheath (introducer). Experimental testing will be conducted to validate the results of the finite element model. Once validated, the modeling approach of the 7-French Durata ICD lead soft-tip will then be applied to the 6F Advanced ICD lead soft-tip model. 


\section{CHAPTER 3 METHODS AND MATERIALS}

\subsection{GENERAL REMARKS}

This chapter discusses the analysis of two soft-tip models with the material properties of MED-4860, a liquid silicone rubber by NuSil Silicone Technology. Figure 3-1 is a flowchart describing the basic path of the modeling and simulation methodology that will be used in the investigation of the soft-tip simulations. The soft-tips were modeled and analyzed using the FEA software ABAQUS v6.8-1. The first soft-tip model was of the 7F Durata ICD Lead Soft-Tip, a St. Jude Medical ICD lead currently in production. The Durata has a 7-French lead body diameter that utilizes a soft-tip with an 8-French diameter. The second soft-tip model is of the 6F Advanced ICD Lead, a St. Jude Medical ICD prototype lead. The Advanced ICD has a 6-French lead body diameter with a 7-French soft-tip diameter. 


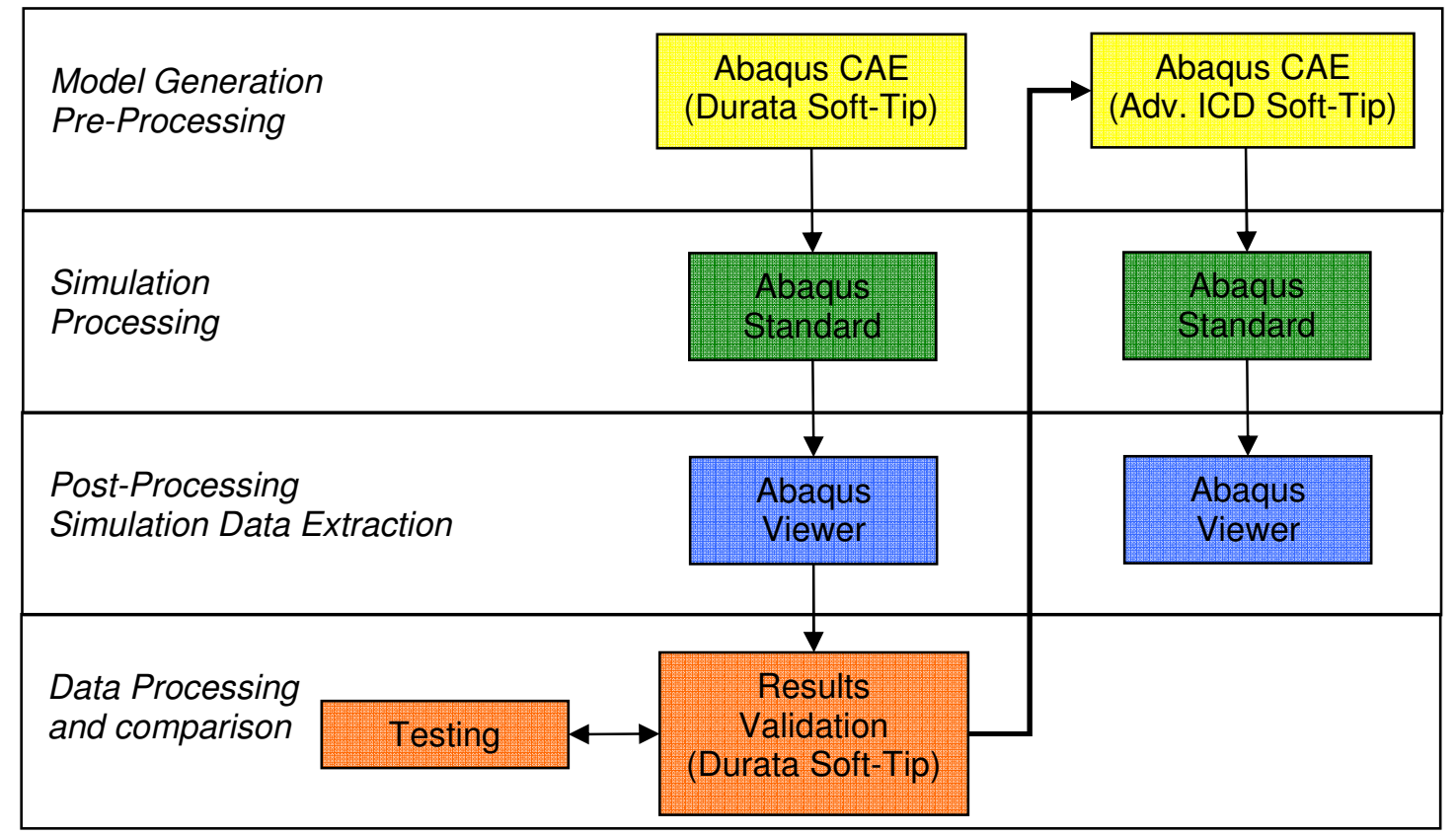

Figure 3-1. Modeling and simulation methodology.

\subsection{COMPARISON BETWEEN PHYSICAL AND FEA SOFT-TIPS}

\subsubsection{Basic Assumptions}

For the purpose of this study some basic assumptions were made to simplify the analysis of the soft-tips. The first and possibly most significant assumption is that the soft-tip simulations were carried out using two dimensional, axisymmetric models. The rational is that with the proper methodology and verification, axisymmetric modeling can significantly decrease processing time and yield stable and accurate results. By reducing the geometry to a 2D axisymmetric model, some of the features of the soft-tip, such as the nubs at the distal tip were ignored. Other simplifications included removing the insert-molded marker band and replacing it with rigid surfaces at the interface. Such simplifications are acceptable because they are away from critical regions or do not significantly contribute to the overall stresses. 
In order to accurately predict multi-axial states of stress for hyperelastic materials, several modes of deformation (i.e. uniaxial tension, planar tension, and equi-biaxial extension) are preferred. Unfortunately, as is typically the case, only uniaxial tension test data was available at the time which may have an effect on the accuracy of the results. Curve fitting will be used to determine the strain energy potential that best fits the test data.

Due to the limited data available, the Poisson's ratio had to be estimated. As an incompressible or nearly incompressible material, rubber has a Poisson's ratio that ranges from 0.49 to 0.50 [23]. For this study a nearly incompressible Poisson's ratio of 0.49 was used for all the models.

Critical to this analysis are the effects of friction at the interface between the silicone soft-tips and the polyethylene introducer. Due to the difficulties in obtaining material samples suitable for friction testing the author researched other friction tests conducted at St. Jude Medical. One test in particular, "Frictional Forces Acting between an Introducer and Silicone Rubber", was conducted at St. Jude Medical and sought to quantify the static and kinetic friction coefficients between introducer material and silicone rubber in wet and dry conditions. In regards to the contact between the soft-tip and introducer, it is assumed that the polyethylene introducer is much stiffer than the silicone soft-tip and therefore has been modeled as an analytical rigid surface. 
The conceptual/FEA model assumes complete fusion between the soft-tip and the metal marker band, with no chance of separation between the components. In reality, there exist a maximum load in which delamination begins to occur.

Although Finite Element Modeling (FEM) is a powerful tool utilized in the design process to minimize development time and cost, the results are only approximations that are ultimately dependant on a single, idealized case. The FEM does not take into account physician dependant variables such as the rate in which a physician pushes a lead through an introducer, and therefore the models presented in this study are limited to quasi-static simulations.

\subsubsection{Hyperelastic Material Models}

St. Jude Medical employs a liquid silicone rubber to over-mold onto a platinum marker band. Rubber is classified as a hyperelastic material, where the stress-strain curve is noted as being non-linearly elastic, isotropic, incompressible, and independent of the strain rate. Figure 3-2 shows a typical stress strain curve for rubber under repeat loading and unloading. 


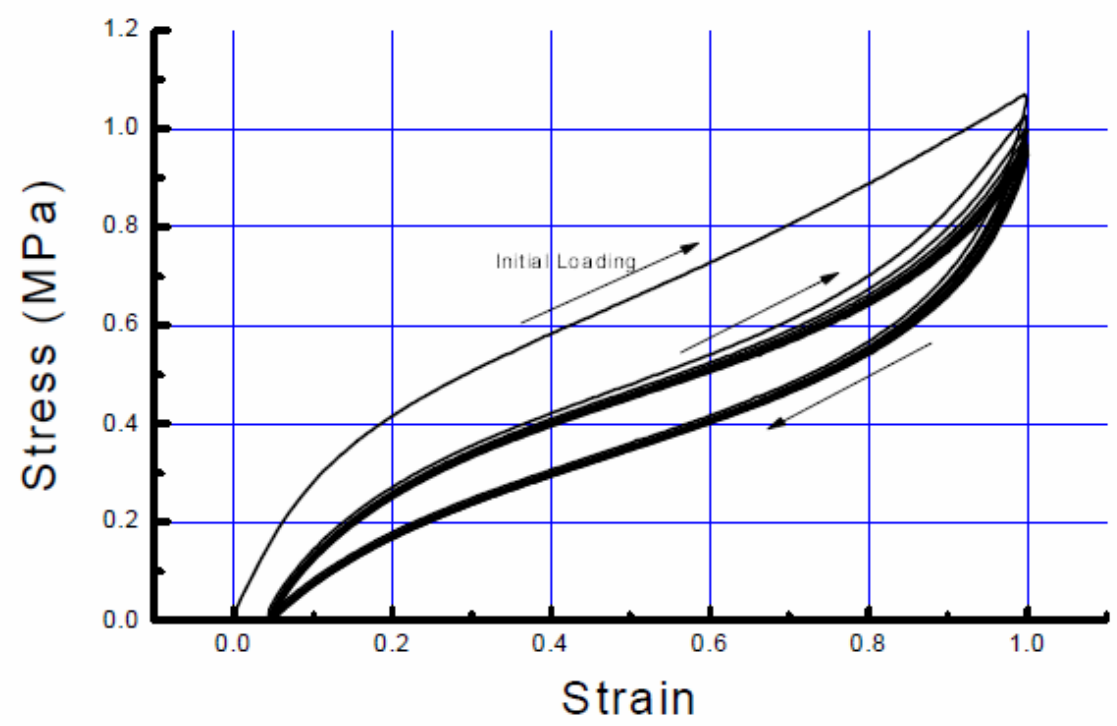

Figure 3-2. Repeat Loading and Unloading [19]

Hyperelastic materials are a special case of Cauchy elastic materials and are commonly referred to as Cauchy-Green elastic materials. A Cauchy elastic material is an elastic simple material where the "state of stress in the current configuration is determined solely by the state of deformation of this current configuration relative to an arbitrary choice of reference configuration" [20]. In other words, the Cauchy stress is independent of the path of deformation. Unlike hyperelastic materials, the work done by the Cauchy stress is dependant on the deformation path.

Hyperelastic materials experience large strains and deformations, and much research has been done to develop material models capable of predicting the non-linear stress-strain behavior typical of hyperelastic materials. There are two fundamental types of material models, incompressible and compressible. Incompressible or nearly incompressible materials, such as rubber, exhibit little to no volumetric change after 
deformation. Solid rubber models can also be classified into two basic categories, physically motivated models and models based on phenomenological theory.

Physically motivated models base the material response on the underlying microstructure and idealize rubber as long chains of cross-linked molecules. Phenomenological models are based on continuum mechanics and base their response on observed stress-strain behavior without considering the microstructure. There are several hyperelastic strain energy potentials available in Abaqus, both physically motivated and phenomenologically based, of which the Marlow form, Arruda-Boyce form and Ogden form material models were considered.

\subsubsection{Material Property Input}

The mechanical properties of Med-4860 were obtained by NuSil Technology's

Testing Services. The sample employed in this test was cut using Die C according to ASTM Standard D412 for Uniaxial Tension Testing of vulcanized rubbers. ASTM D412 specifies a dog-bone shaped specimen. Die $\mathrm{C}$ has an overall length of 4.5 inches with a narrow section 1.31 inches long. This provides a gauge length of 1 inch long and a gauge width of 0.25 inch.

Shown in Figure 3-3 and 3-4 is a standard setup for Uniaxial Tension and the state of stress in this configuration, respectively [19]. This setup allows operator to measure strain only in the region where a uniform state of strain exists. By utilizing non-contact measurement systems such as laser and video extensometers, measurements can be obtained without interfering with the response. 


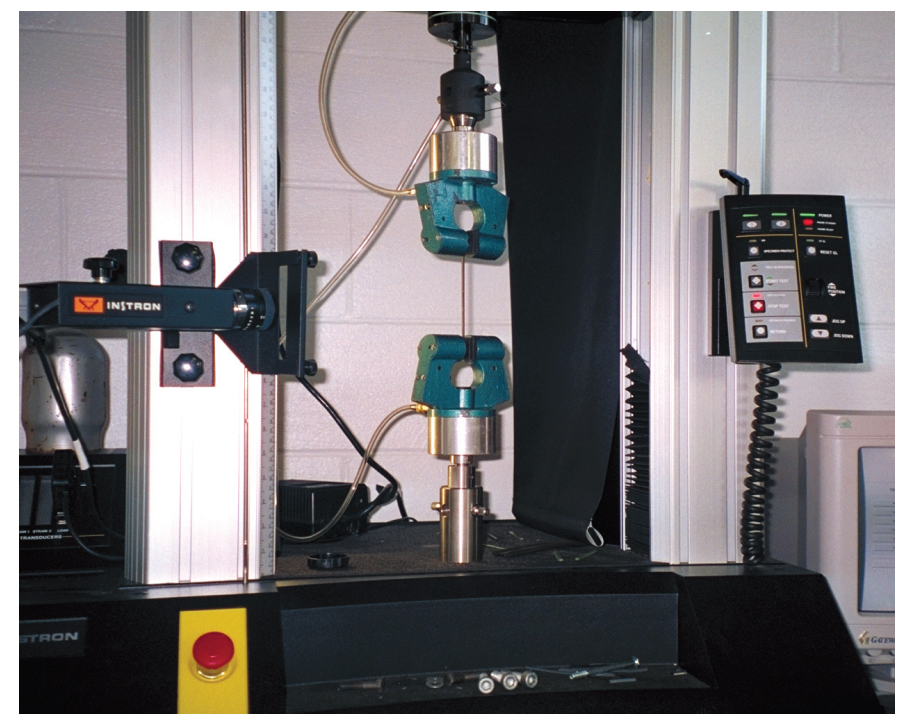

Figure 3-3. A tension experiment using a video extensometer.

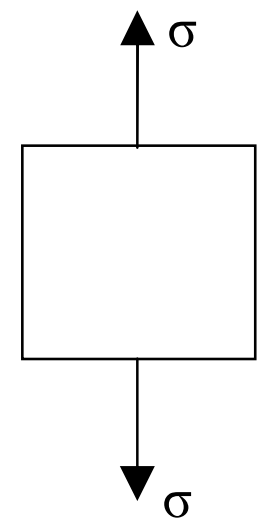

Figure 3-4. State of stress of uniaxial tensile test.

The physical properties of Med-4860 are shown in Table 3-1.

Table 3-1. Physical properties of Med-4860.

\begin{tabular}{|l|c|}
\hline Specific Gravity & 1.16 \\
\hline Durometer, Type A & 62 \\
\hline Tensile Strength, psi & 1200 \\
\hline Elongation, \% & 500 \\
\hline Tear Strength, ppi / kN/m & 48.2 \\
\hline Stress @ 200\%,psi & 650 \\
\hline
\end{tabular}


The uniaxial test data was input into the model. Abaqus provides automatic curve fitting tools to determine a strain energy potential that best fits the experimental data. The evaluation process of a basic unit cube data-check is outlined in Figure 3-5.

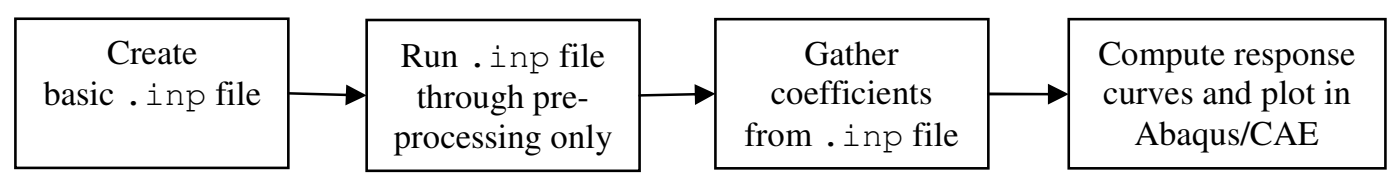

Figure 3-5. Automatic material evaluation procedure in Abaqus.

The results of the curve fitting analysis showed that all three strain energy potentials have good correlation to the test data (Figure 3-6). In order to minimize the error, the average root mean square error was used to calculate the precision between the theoretical stress and the measured stress.

$$
\operatorname{Avg} R M S E=\frac{1}{M} \sqrt{\sum_{i=1}^{N}\left(\frac{\sigma_{\exp }-\sigma_{F E A}}{\sigma_{\exp }}\right)}
$$

Equation 1

where: $\sigma_{\text {exp }}$ is the measured engineering stress,

$\sigma_{\mathrm{FEA}}$ is the theoretical stress,

$M \quad$ is the total number of points where stress was calculated by FEA and by experimental measurement 


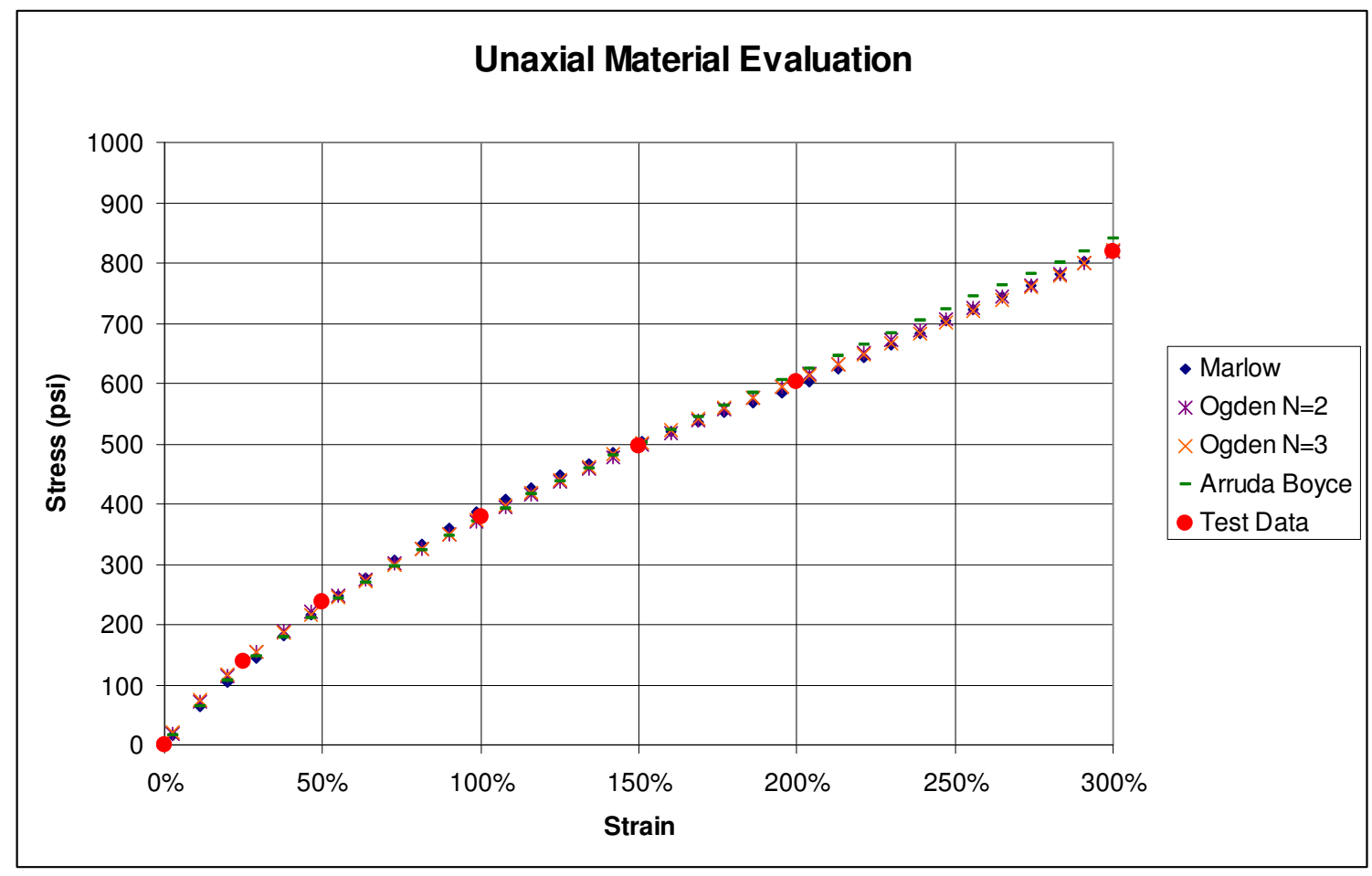

Figure 3-6. Stress-Strain plots for uniaxial response.

Four hyperelastic strain energy potentials were considered for this project, of which the best fit was the Ogden form, second order model with 3.39\% average error.

\subsection{MODELING OF THE 7F DURATA ICD LEAD SOFT-TIP}

Abaqus CAE v6.8-1 was used to generate the 2D, Axisymmetric model of the Durata 7-French Soft-Tip and 7F Peel-Away Sheath (i.e. introducer). The model was validated against FDA-approved experimental introducer tests conducted at St. Jude Medical which measure the force required to push a lead through an introducer. The geometry of the simplified soft-tip and introducer is shown in Figures 3-7 and 3-8, respectively. The body of the Durata soft-tip is 7-French (7F $=0.092$ inches) in diameter with an 8-French $(8 \mathrm{~F}=0.105$ inches $)$ flange. 

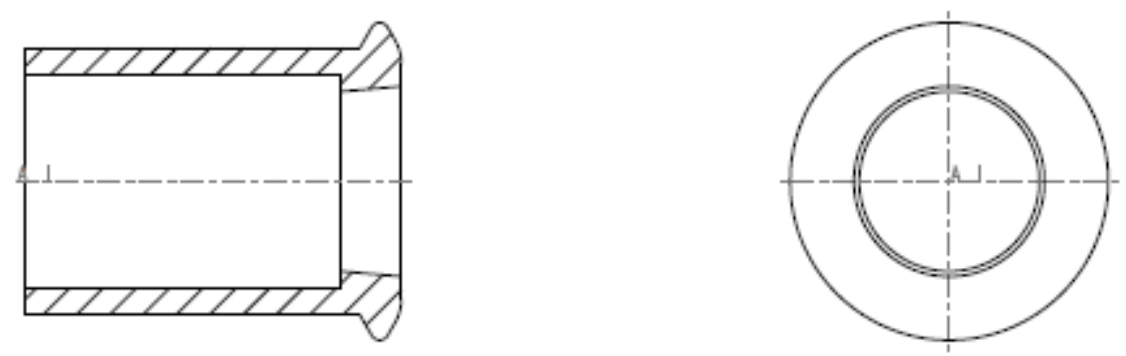

Figure 3-7. Geometry of the SJM 7F Durata ICD Soft-Tip.

The outer dimensions of the over-molded soft-tip were verified with a digital microscope. The inner diameter of the soft-tip is constrained by the marker band. The introducer dimensions were based on gage measurements from a physical 7F Peel-Away Sheath (introducer). The total length of the introducer was reduced from eight inches to three inches.

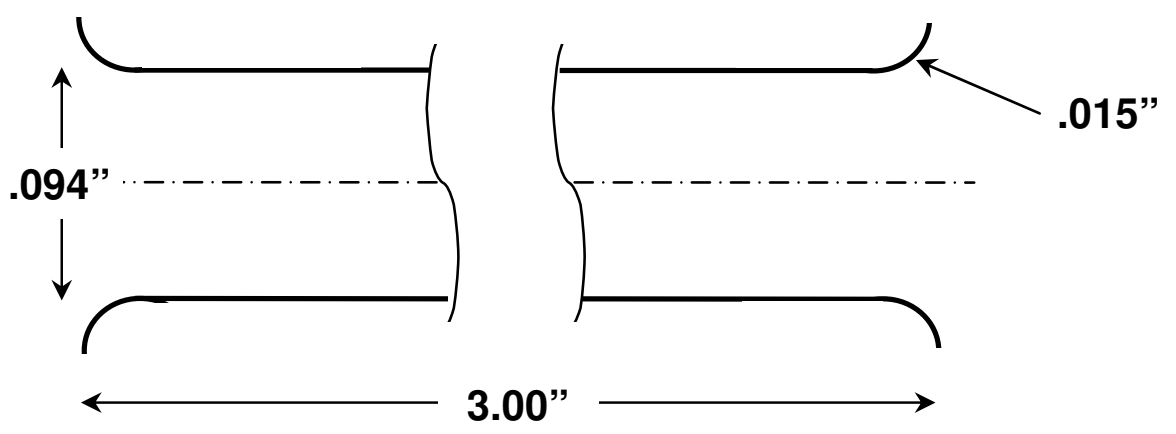

Figure 3-8. Simplified 7-French Peel-Away Sheath.

\subsubsection{Meshing of the Durata}

The finite element mesh in Figure 3-9 is of the 7F Durata Soft-Tip was modeled as a 2D Axisymmetric deformable mesh consisting of 4-noded hybrid axisymmetric 
elements with reduced integration and enhanced hourglass control (CAX4RH). The average edge length of the elements was $7.45 \mathrm{e}^{-4}$ inches, resulting in 2,099 linear quadrilateral elements. This Durata model has an average aspect ratio of 1.09 with the worst being 2.18. The introducer was modeled as an analytical rigid surface and there required no meshing.

Figure 3-9. Mesh of the SJM 7F Durata Soft-Tip.

\subsubsection{Loading and Boundary Conditions of the Durata and 7F Introducer}

Each node of the axisymmetric elements has six degrees of freedom, translation in the $\mathrm{x}, \mathrm{y}$, and $\mathrm{z}$-directions along with rotations about the $\mathrm{x}, \mathrm{y}$, and $\mathrm{z}$-axis. To define the marker band which is essentially bonded to the soft-tip during the over-mold process, the nodes at this soft-tip to marker band interface (Figure 3-10) were fixed for all degrees of freedom and propagated throughout the simulation. The introducer was allowed to 
translate three inches along the Y-axis. All other degrees of freedom were fixed on the introducer.

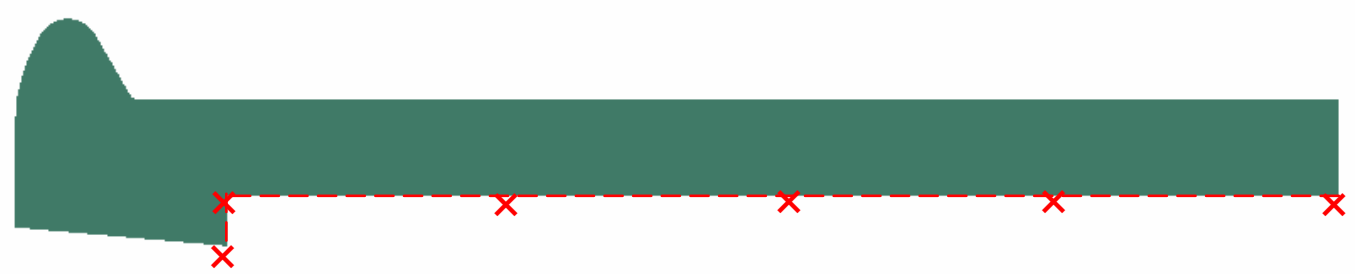

Figure 3-10. Boundary conditions at soft-tip/marker band interface of Durata model.

\subsubsection{Interaction Properties}

A surface to surface contact was created to define the interaction between the introducer and the soft-tip. The introducer was chosen as the master surface with the outer diameter of the soft-tip being the slave surface. Abaqus provides a Coulomb friction model that allows the user to specify the static and kinetic friction coefficients directly (Equation 2).

$$
\mu=\mu_{k}+\left(\mu_{s}-\mu_{k}\right) e^{-d_{c} \dot{\gamma}_{e q}},
$$

where: $\quad \mu_{k}$ is the kinetic friction coefficient,

$\mu_{s}$ is the static friction coefficient,

$d_{c}$ is the user-defined decay coefficient, and

$\dot{\gamma}_{e q}$ is the slip rate 
With this model, the friction coefficients between the soft-tip and introducer were defined as 0.37 and 0.27 , for the static and kinetic friction, respectively. Figure 3-11 shows a plot of the slip rate versus friction with a decay coefficient of 0.01 .

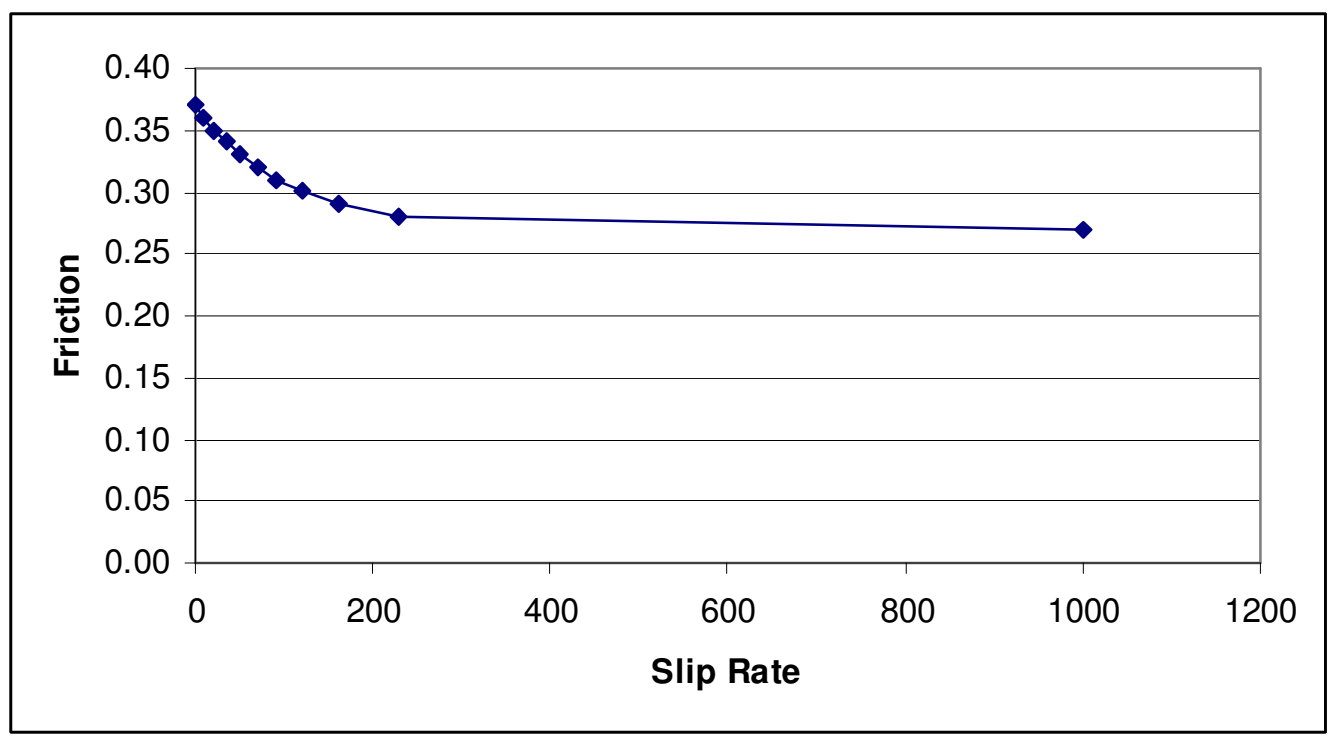

Figure 3-11. Exponential decay friction model specified with test data points.

Table 3-2 shows the parameters in detail that were used to define the interaction between the Durata soft-tip and the introducer.

Table 3-2. Interaction properties between the Soft-Tip and Introducer.

\begin{tabular}{|l|c|}
\hline \multicolumn{2}{|c|}{ Soft-Tip/Introducer Interaction } \\
\hline Master Surface & Introducer \\
\hline Slave Surface & Soft-Tip \\
\hline Discretization Method & Surface-to-Surface \\
\hline Sliding Formulation & Finite Sliding \\
\hline Contact Tracking & Single Configuration \\
\hline \multicolumn{2}{|c|}{ Interaction Property } \\
\hline Friction Formulation & Static-Kinetic Exponential Decay \\
\hline Pressure-Overclosure & Exponential \\
\hline
\end{tabular}


The surface constraints of the introducer and Durata soft-tip are shown in Figure 3-12. A rigid body constraint was used to couple the introducer's analytical surface to its reference node. For the soft-tip a surface-based, kinematic coupling constraint was used to couple the soft-tip reference node to the coupling nodes at the bonding interface of the over-molded soft-tip. The coupling constraint allows a group of coupling nodes to be constrained to the rigid body motion of a single reference node. The soft-tip reference node was also used to capture the reaction force as the introducer slides across the soft-tip. The reaction force correlates to the "push force" in the experimental introducer test.

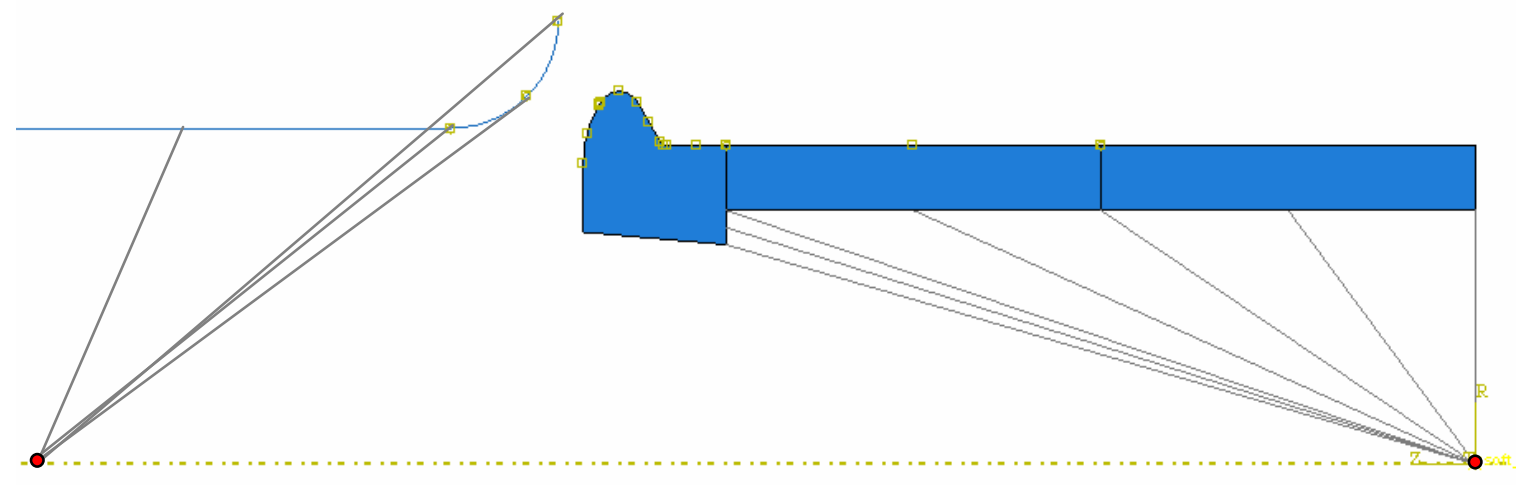

Figure 3-12. Surface constraints of Introducer and Durata Soft-Tip.

Due to the large deformation of the soft-tip flange as it passes through the introducer, a self-contact interaction (Figure 3-13) was implemented between outer surface of the soft-tip flange and the outer surface of the soft-tip body. 


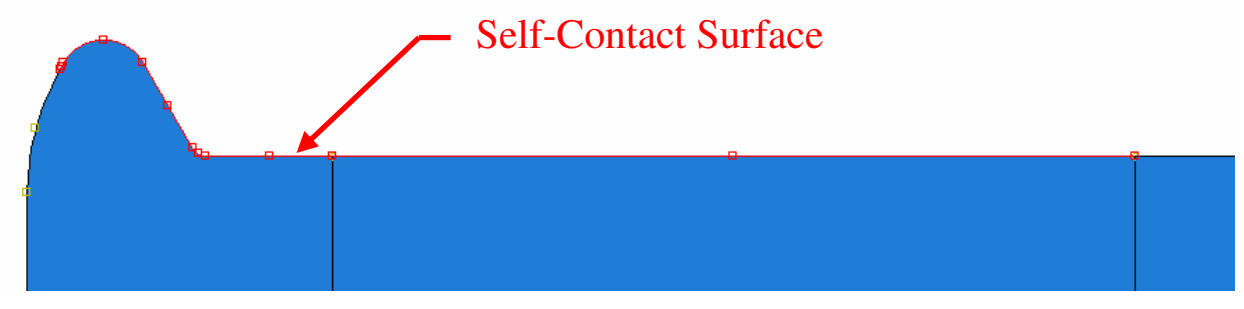

Figure 3-13. Self-Contact surface of the SJM Durata soft-tip.

\subsection{MODELING OF THE 6F ADVANCED ICD LEAD SOFT-TIP}

Modeling of the $6 \mathrm{~F}$ Advanced ICD soft-tip was similar to that of the Durata softtip. The general dimensions of the simplified 2D, Axisymmetric model of the $6 \mathrm{~F}$ Advanced ICD Soft-Tip and 6F Peel-Away Sheath (i.e. introducer) are shown in Figures 3-14 and 3-15, respectively. The model was validated against an experimental introducer tests conducted at St. Jude Medical. The body of the Advaced ICD soft-tip is 6-French $(6 \mathrm{~F}=0.079$ inches $)$ in diameter with a 7-French $(7 \mathrm{~F}=0.092$ inches $)$ flange.
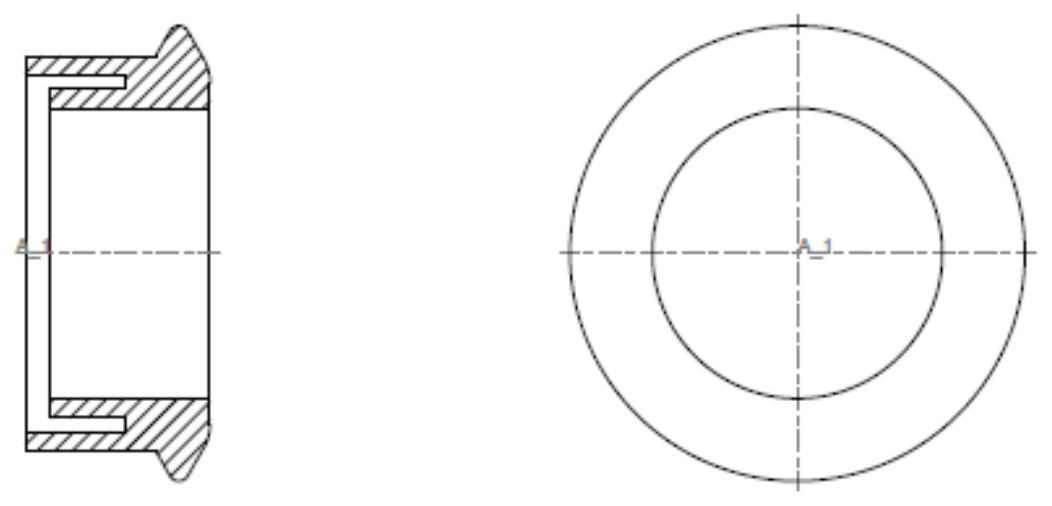

Figure 3-14. Geometry of the 6F Advanced ICD Soft-Tip. 
The outer and inner dimensions of the over-molded soft-tip were verified with a digital microscope, while the insert-molded marker band controls the dimensions of the step inside the soft-tip. The introducer dimensions in Figure 3-15 were based on gage measurements from a physical 6F Peel-Away Sheath (introducer). The total introducer length of three inches remained the same from the $7 \mathrm{~F}$ to $6 \mathrm{~F}$ introducer.

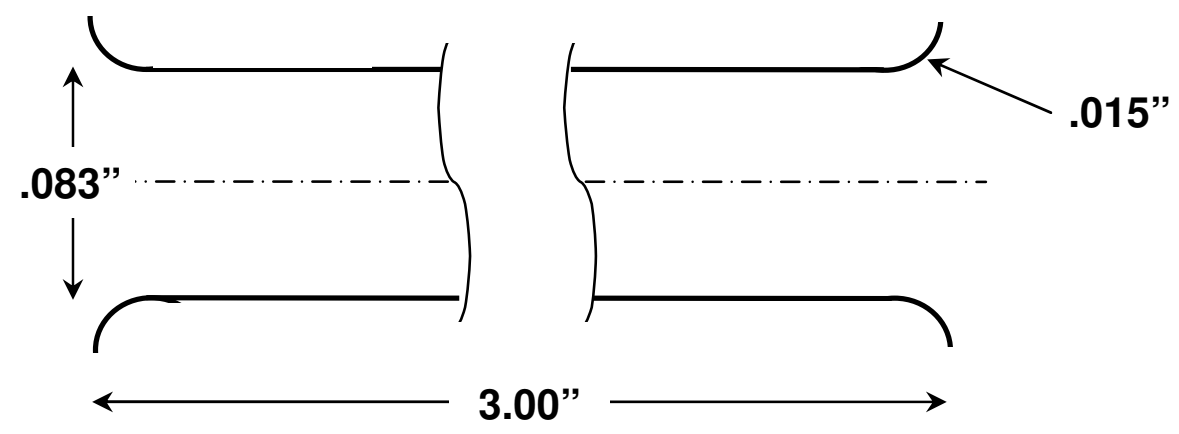

Figure 3-15. Simplified 6-French Peel-Away Sheath.

\subsubsection{Meshing of the Advanced ICD}

The modeling approach of the $6 \mathrm{~F}$ Advanced ICD was similar to that of the $7 \mathrm{~F}$ Durata - 2D Axisymmetric deformable mesh consisting of 4-noded hybrid axisymmetric elements with reduced integration and enhanced hourglass control (CAX4RH). The average edge length of the elements was $6.20 \mathrm{e}^{-4}$ inches, resulting in 691 linear quadrilateral elements. The Advanced ICD mesh (Figure 3-16) has an average aspect ratio of 1.28 with the worst being 2.26 . The $6 \mathrm{~F}$ introducer was modeled as an analytical rigid surface and there required no meshing. 


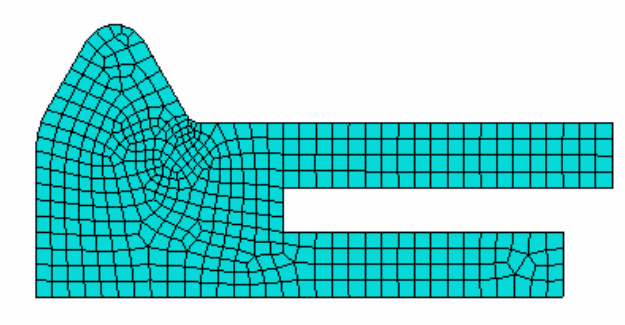

Figure 3-16. Mesh of the 6F Advaced ICD Soft-Tip.

\subsubsection{Loading and Boundary Conditions of the Advanced ICD and 6F Introducer \\ The nodes at this soft-tip to marker band interface (Figure 3-17) were fixed for all \\ degrees of freedom and propagated throughout the simulation. The introducer was allowed to translate three inches along the Y-axis. All other degrees of freedom were fixed on the introducer.}




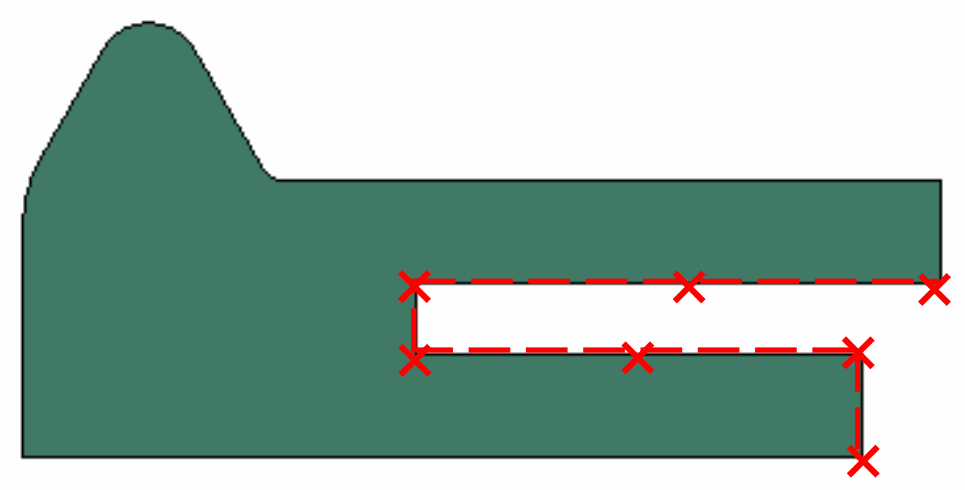

Figure 3-17. Boundary conditions of Advanced ICD model at soft-tip/marker band interface.

\subsubsection{Interaction Properties}

A surface to surface contact was created to define the interaction between the introducer and the soft-tip. The introducer was chosen as the master surface with the outer diameter of the soft-tip being the slave surface. The friction coefficients between the soft-tip and introducer were carried over from the Durata simulation as 0.37 and 0.27 , for the static and kinetic friction, respectively.

Table 3-3 shows the parameters in detail that were used to define the interaction between the Durata soft-tip and the introducer. 
Table 3-3. Interaction properties between the Soft-Tip and Introducer.

\begin{tabular}{|l|c|}
\hline \multicolumn{2}{|c|}{ Soft-Tip/Introducer Interaction } \\
\hline Master Surface & Introducer \\
\hline Slave Surface & Soft-Tip \\
\hline Discretization Method & Surface-to-Surface \\
\hline Sliding Formulation & Finite Sliding \\
\hline Contact Tracking & Single Configuration \\
\hline \multicolumn{2}{|c|}{ Interaction Property } \\
\hline Friction Formulation & Static-Kinetic Exponential Decay \\
\hline Pressure-Overclosure & Exponential \\
\hline
\end{tabular}

The surface constraints of the introducer and Advanced ICD soft-tip are shown in Figure 3-18. A rigid body constraint was used to couple the introducer's analytical surface to its reference node. For the soft-tip a surface-based, kinematic coupling constraint was used to couple the soft-tip reference node to the coupling nodes at the bonding interface of the insert-molded soft-tip. Figure 3-19 shows the self-contact surface interaction between the soft-tip body and flange. 


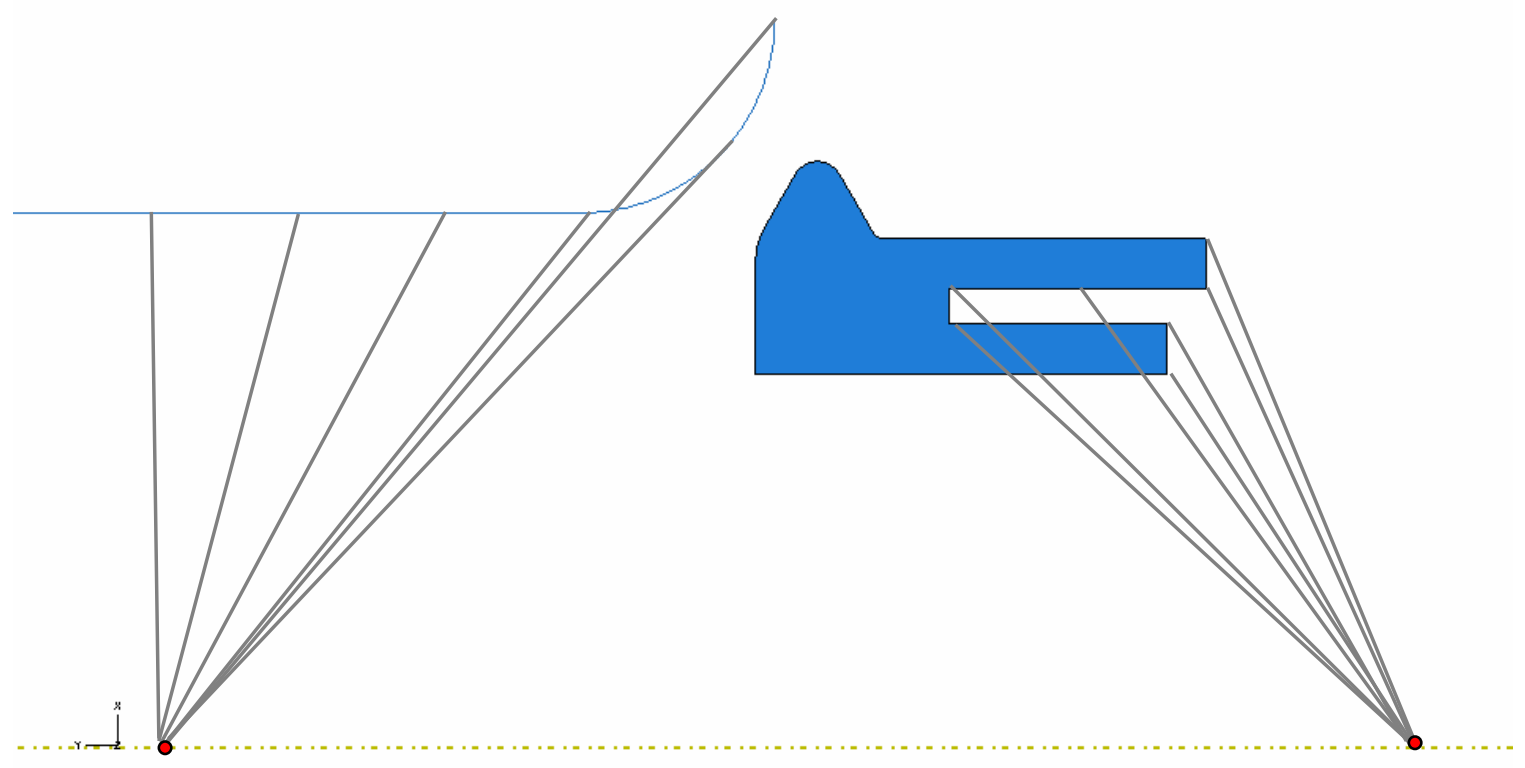

Figure 3-18. Surface constraints of Introducer and Advanced ICD Soft-Tip.

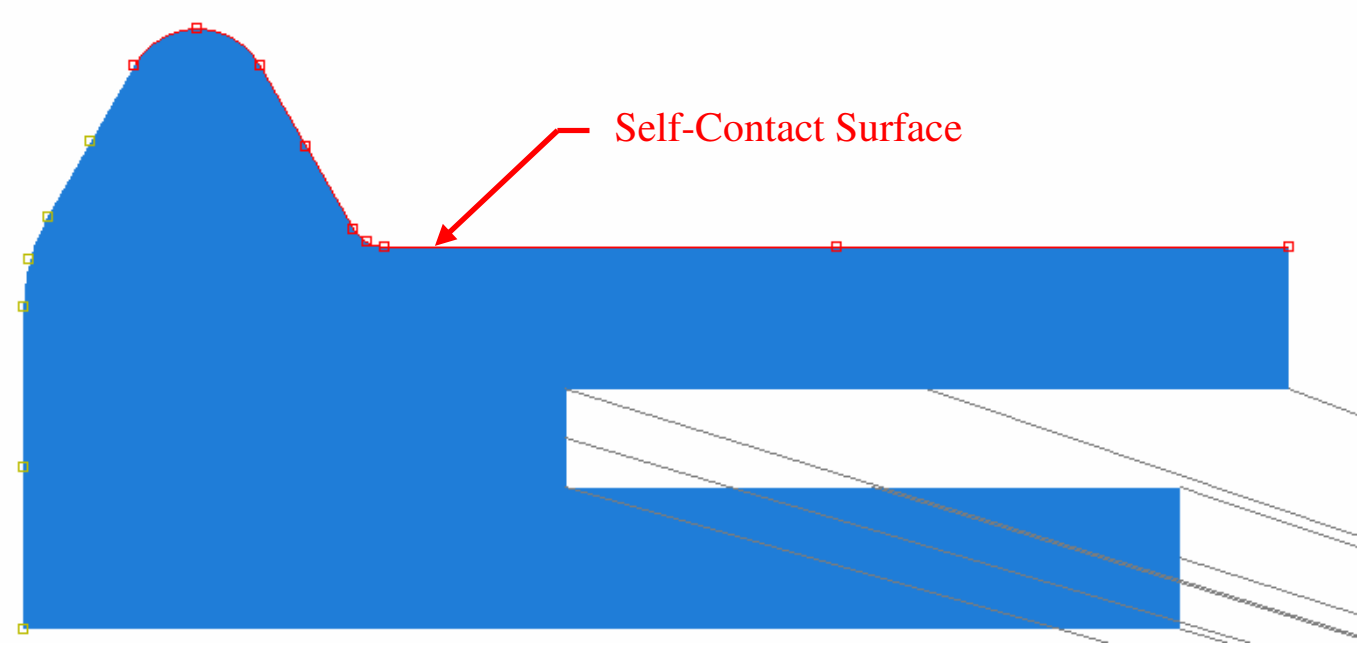

Figure 3-19. Self-Contact surface of Advanced ICD Soft-Tip. 


\subsection{MESH CONVERGENCE}

A mesh convergence study is important in any finite element analysis to ensure that the results of an analysis are not affected by changing the size of a mesh. The mesh convergence studies in this thesis consisted of performing a mesh refinement on the Durata and Advanced ICD Soft-Tip models, and evaluating two variables for each model - change in maximum push force and percent difference from experimental data. The mesh for each model was deemed converged when changes in the maximum push force were less than $5 \%$. The parameters of the mesh refinement study are recorded in Tables 3-4 and 3-5 for the Durata and Advanced ICD Soft-Tips, respectively.

Table 3-4. Summary of mesh refinement for Durata Soft-Tip model.

\begin{tabular}{|l|c|c|c|c|}
\hline Lead Type & $\begin{array}{c}\text { Seeding } \\
\text { Ratio }\end{array}$ & $\begin{array}{c}\text { \# of } \\
\text { Elements }\end{array}$ & $\begin{array}{c}\text { Element } \\
\text { Type }\end{array}$ & $\begin{array}{c}\text { \# of } \\
\text { Nodes }\end{array}$ \\
\hline 7F Durata & 0.0006 & 3029 & CAX4RH & 4069 \\
\hline 7F Durata & 0.0008 & 2099 & CAX4RH & 2279 \\
\hline 7F Durata & 0.0010 & 1380 & CAX4RH & 1525 \\
\hline 7F Durata & 0.0012 & 1055 & CAX4RH & 1177 \\
\hline 7F Durata & 0.0014 & 706 & CAX4RH & 812 \\
\hline 7F Durata & 0.0016 & 586 & CAX4RH & 680 \\
\hline 7F Durata & 0.0018 & 447 & CAX4RH & 531 \\
\hline 7F Durata & 0.0020 & 372 & CAX4RH & 446 \\
\hline
\end{tabular}

Table 3-5. Summary of mesh refinement for Advanced ICD Soft-Tip model.

\begin{tabular}{|c|c|c|c|c|}
\hline Lead Type & $\begin{array}{c}\text { Seeding } \\
\text { Ratio }\end{array}$ & $\begin{array}{c}\text { \# of } \\
\text { Elements }\end{array}$ & $\begin{array}{c}\text { Element } \\
\text { Type }\end{array}$ & $\begin{array}{c}\text { \# of } \\
\text { Nodes }\end{array}$ \\
\hline 6F Adv ICD & 0.0008 & 691 & CAX4RH & 774 \\
\hline 6F Adv ICD & 0.0010 & 466 & CAX4RH & 535 \\
\hline 6F Adv ICD & 0.0020 & 98 & CAX4RH & 133 \\
\hline 6F Adv ICD & 0.0025 & 80 & CAX4RH & 112 \\
\hline 6F Adv ICD & 0.0030 & 61 & CAX4RH & 87 \\
\hline
\end{tabular}


The change in maximum push force was plotted against the level of mesh refinement for the Durata and Advanced ICD Soft-Tips. Shown in Figure 3-20, the maximum push force varied less than $3 \%$ with mesh refinement..

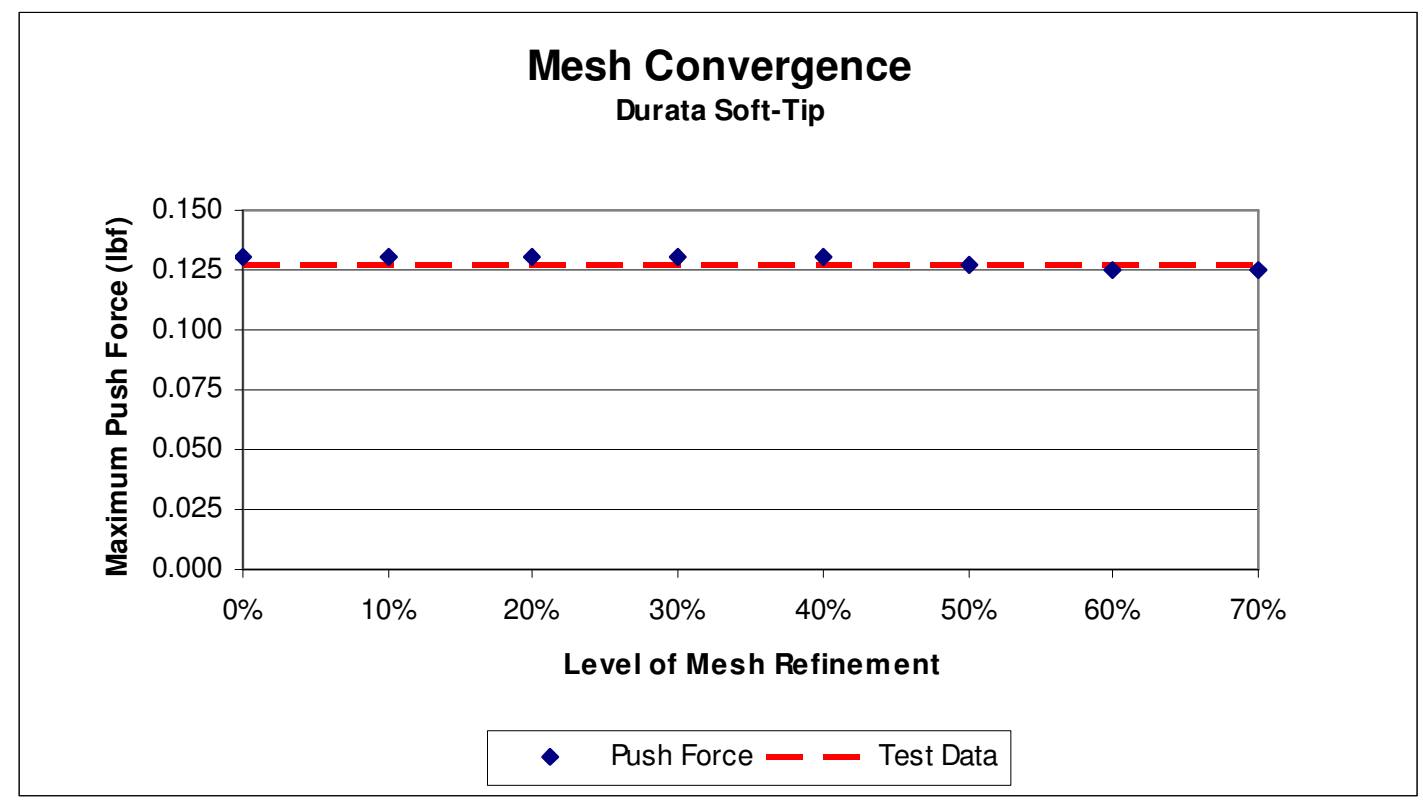

Figure 3-20. Mesh convergence curve for Durata Soft-Tip model.

Mesh refinement had a larger affect on the Advanced ICD Soft-Tip than the Durata Soft-Tip. Figure 3-21 shows the maximum push force stabilized at approximately $33 \%$, where the seeding ratio was 0.0020 . 


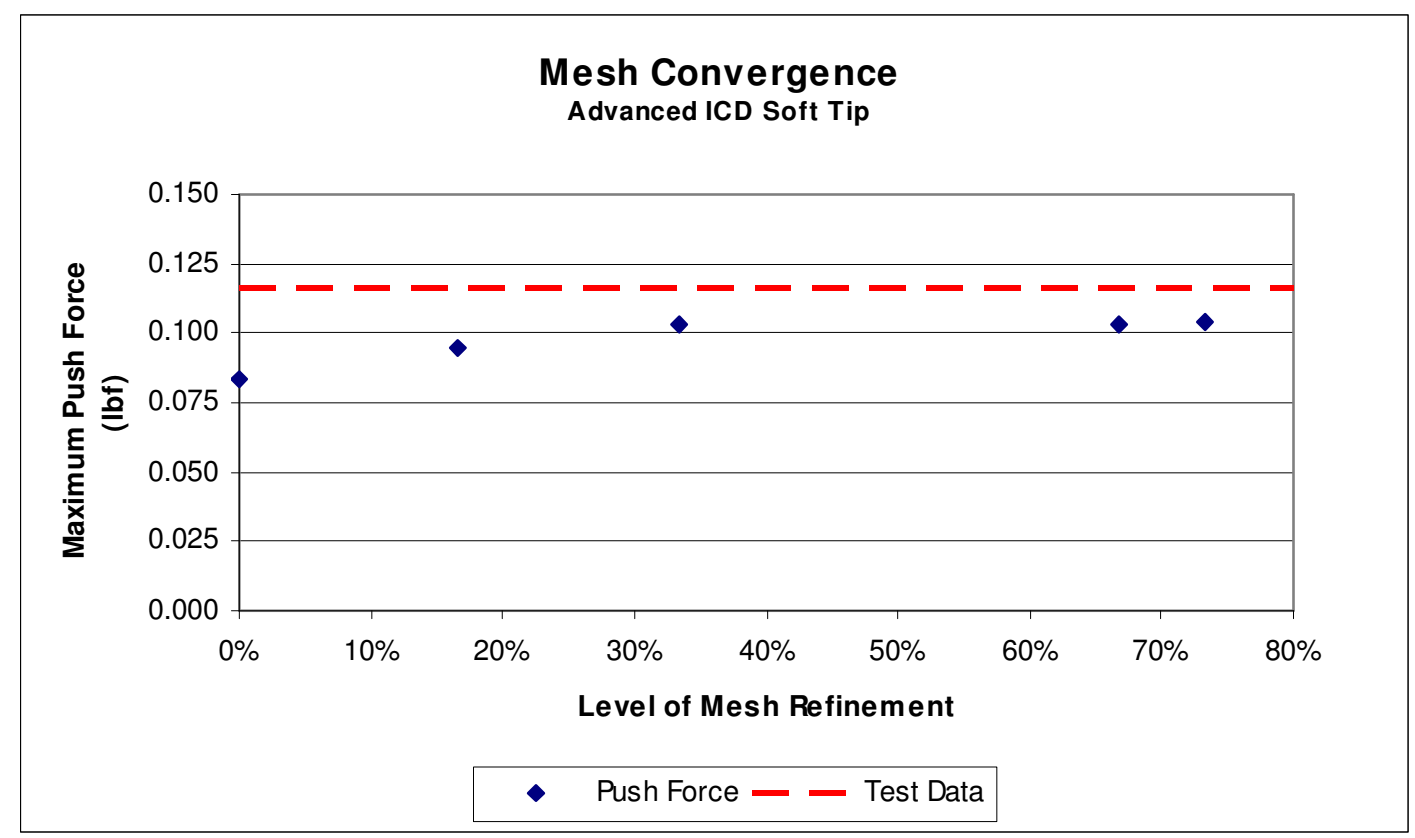

Figure 3-21. Mesh convergence curve for Advanced ICD Soft-Tip model. 


\section{CHAPTER 4}

\section{RESULTS}

\subsection{GENERAL REMARKS}

This chapter describes the results of the FEA on the Durata and Advanced ICD Soft-Tips described in Chapter 3. A comparison between the physical soft-tips and the finite element models are also presented here.

Having developed the finite element model of the Durata Soft-Tip it was necessary to validate the model with a physical introducer test. The results of the validation test for the Durata Soft-Tip are presented in section 4.4. Once the results were deemed acceptable, the same modeling methodology was applied to the Advanced ICD Soft-Tip. The predicted values were then compared to the physical introducer test of the Advanced ICD Soft-Tip and presented in section 4.5.

\subsection{ANALYSIS RESULTS OF THE DURATA SOFT-TIP}

The analysis results in Figure 4-1 show the Von-Mises Stress distribution after the soft-tip has been fully inserted into the 7F introducer. The distal end of the soft-tip is not supported by the marker band, and therefore can collapse into the introducer. The trailing face of the soft-tip flange shows a high stress concentration region where a maximum Von-Mises stress of 273.6psi was recorded at element 84. 

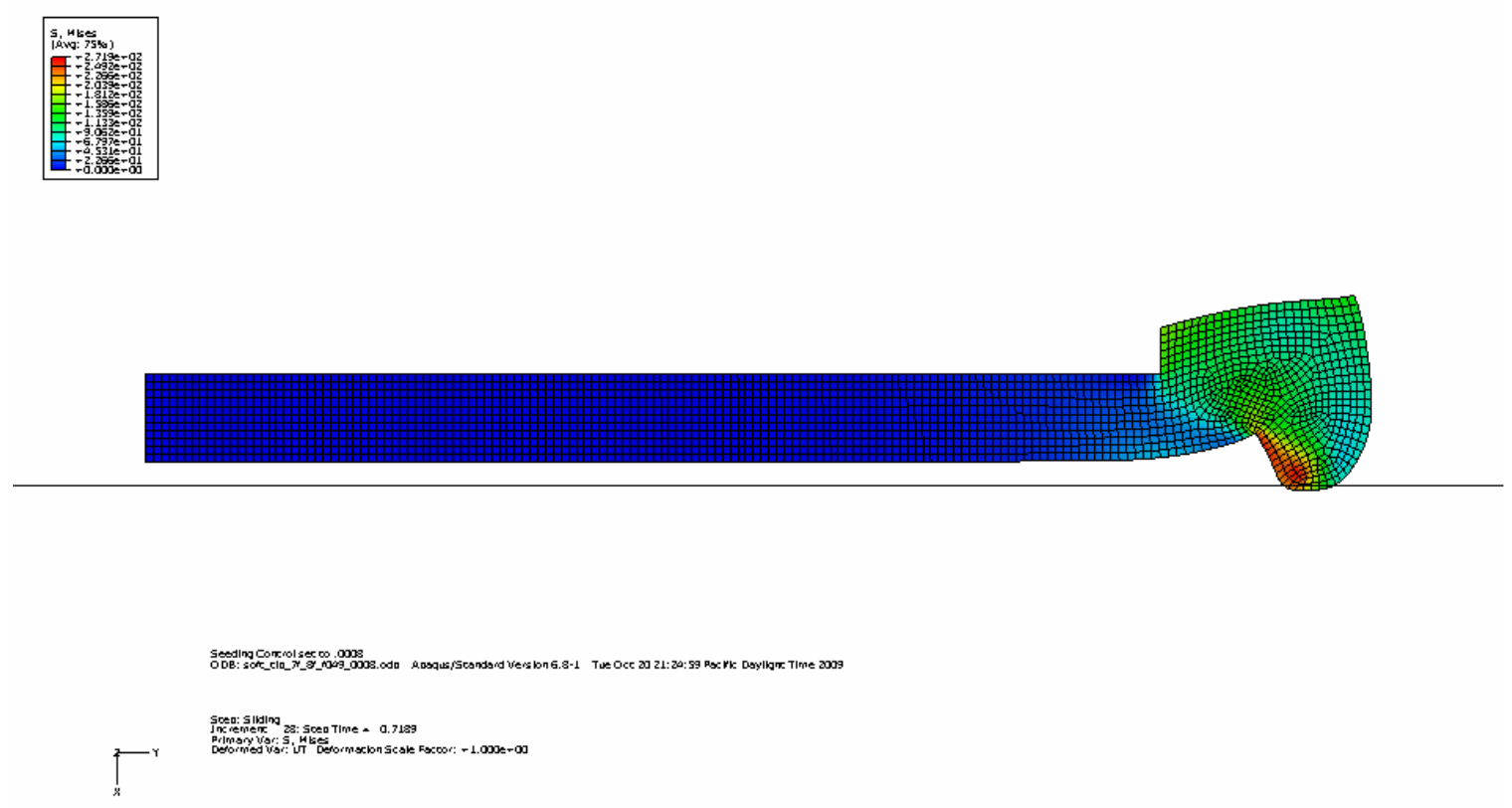

Figure 4-1. Durata Mises stress distribution across the soft-tip at $\mathbf{t}=\mathbf{0 . 7 1 9}$ sec.

Figure 4-2 shows the undeformed soft-tip overlaid onto a contour plot of the deformed soft-tip. When pushed through a 7F introducer the inner diameter of the soft-tip is effectively reduced from 0.065 inches to 0.055 inches - a $15.4 \%$ decrease in diameter.

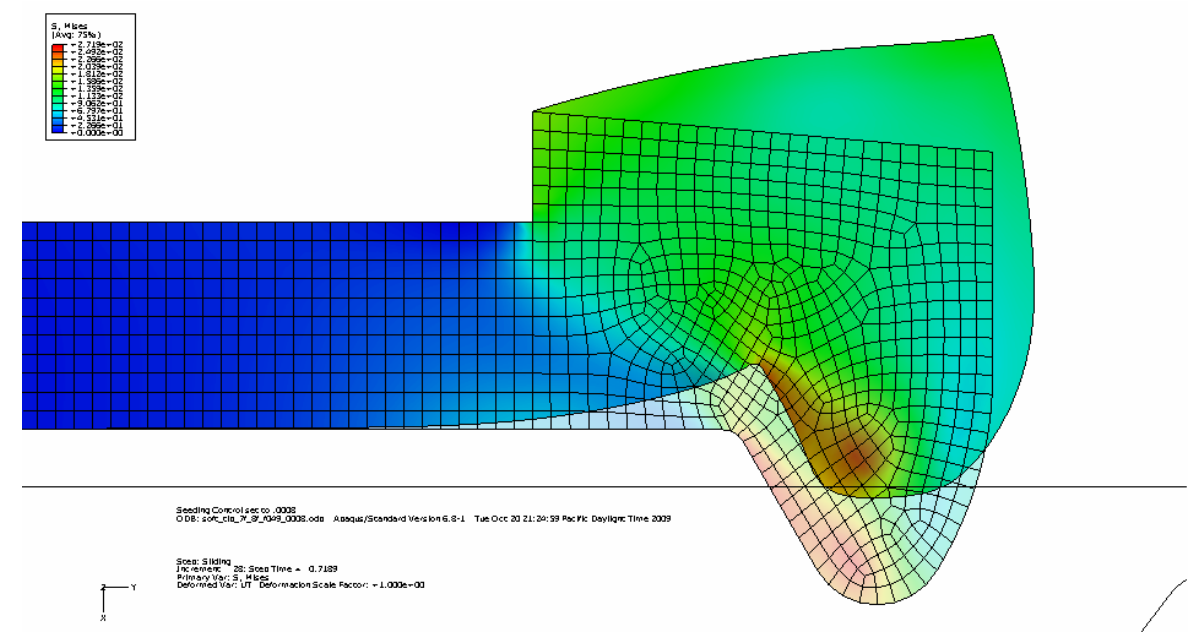

Figure 4-2. Displacement of the Durata soft-tip during insertion. 
As discussed in Section 3.5 of this report, a mesh convergence study was conducted to ensure the resulting push force was not affected by the mesh density. Figure 4-3 shows the difference in the Von-Mises stress distribution when the seeding ratio was increased from 0.0020 to 0.0008 . While the overall deformation and stress distribution was similar for both models, the model with a seeding ratio of 0.0020 had a maximum Mises stress of 251.6psi, compared to the model with a seeding ratio of 0.0008 which had a maximum Mises stress of 273.6psi - an increase of 3.8\%.
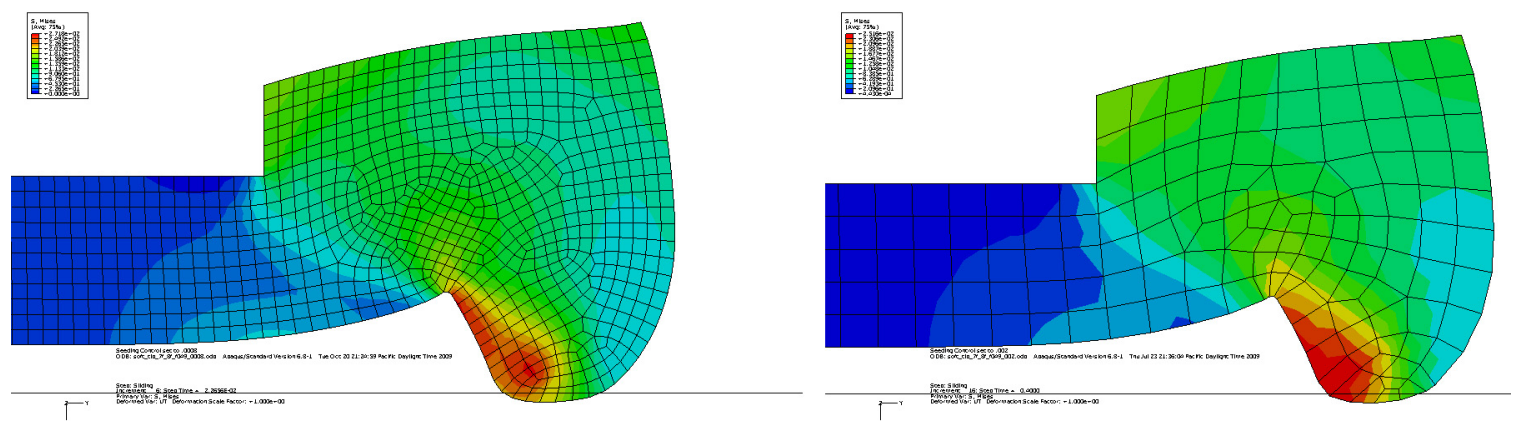

Figure 4-3. Durata Mises stress distributions for 0.0008 and 0.0020 seeding ratios.

The reaction force along the Y-Axis, RF2, was recorded throughout the simulation as the 7F introducer was sliding over the 7F Durata soft-tip and is presented in Figure 4-4. The maximum RF2 equates to the push force recorded during an experimental introducer test. Using a seeding ratio of 0.0008 , the maximum RF2 in this simulation was $0.125 \mathrm{lbf}$. 


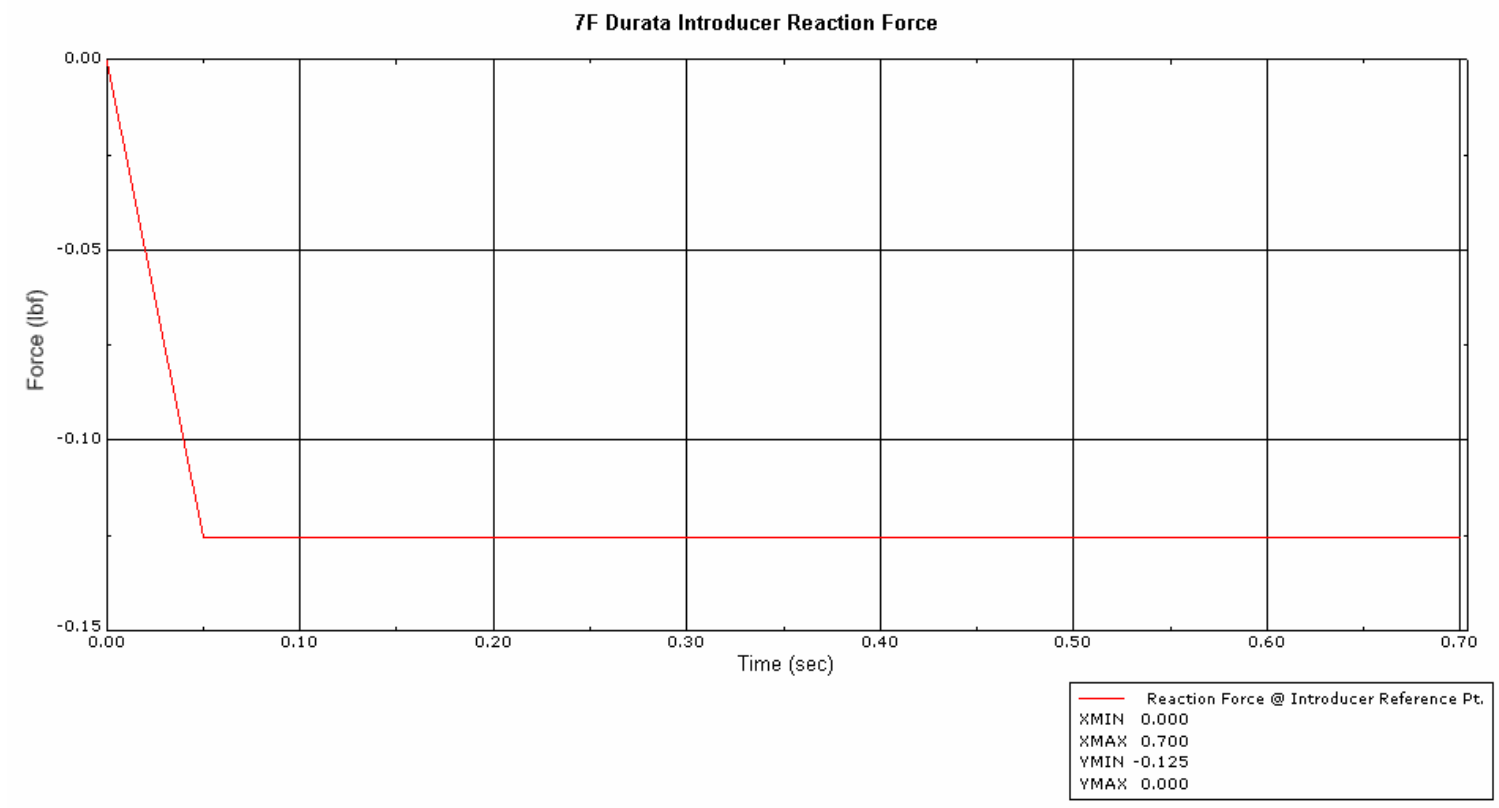

Figure 4-4. Simulated push force of the 7F Durata Soft-Tip and 7F Introducer models.

\subsection{ANALYSIS RESULTS OF THE ADVANCED ICD SOFT-TIP}

The analysis results in Figure 4-5 show the Von-Mises Stress distribution as the 6F Advanced ICD Soft-Tip is being inserted into the $6 \mathrm{~F}$ introducer. The flange of the Advanced ICD Soft-Tip does not collapse inward like the Durata soft-tip, but folds over on itself as it is compressed against the introducer. This creates a stress concentration at the soft-tip body and flange interface. 

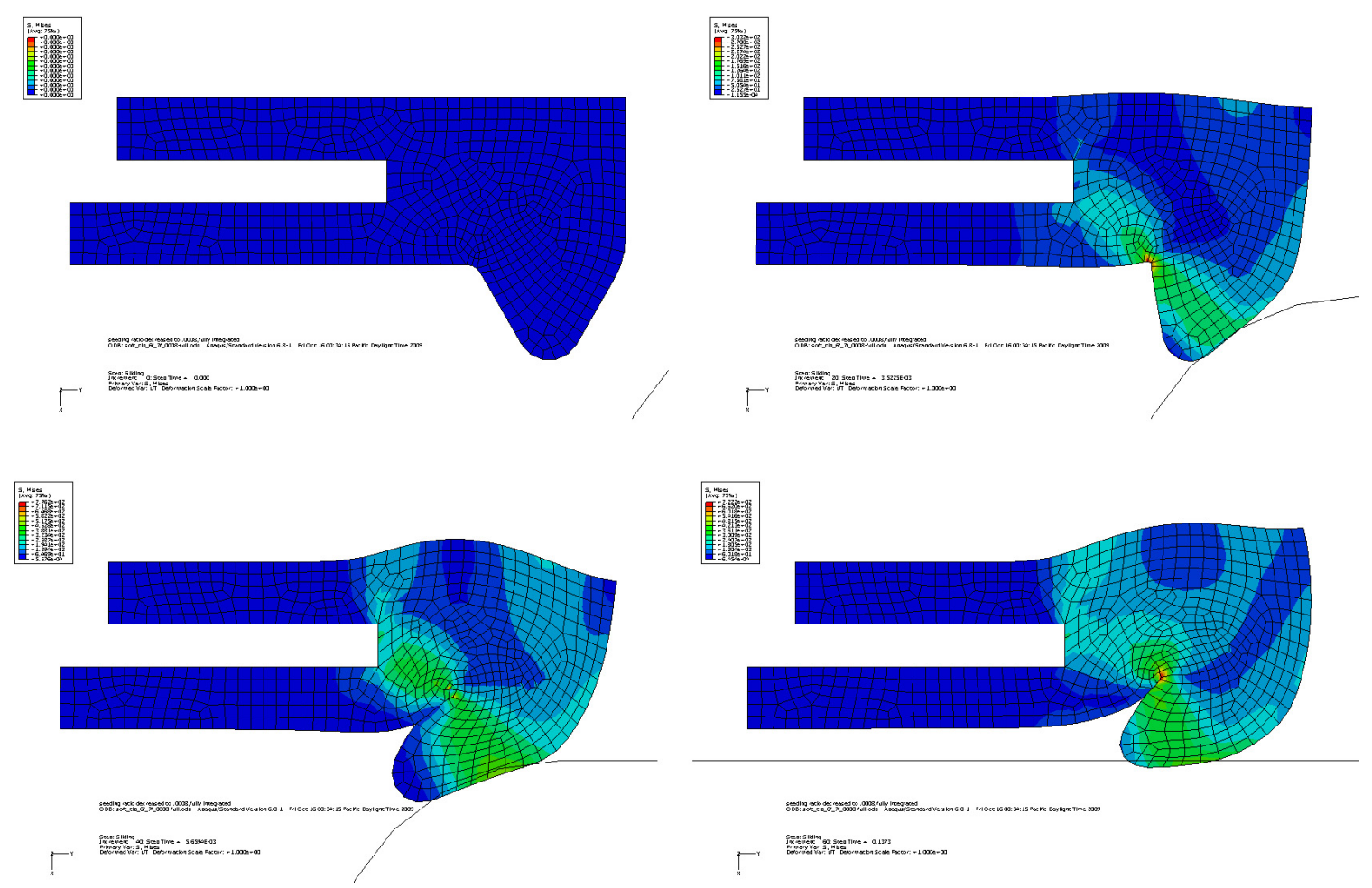

Figure 4-5. Advanced ICD Mises stress distribution at steps 0, 20, 40, and 60.

A maximum Von-Mises stress of $722.2 \mathrm{psi}$ was recorded at the stress concentration between the flange and soft-tip body. Compared to the 7F Durata Soft-Tip the 6F Advanced ICD Soft-Tip experienced an increase in the maximum Von-Mises stress of approximately $164 \%$.

Figure 4-6 shows the undeformed soft-tip overlaid onto a plot of the deformed soft-tip. When pushed through a $6 \mathrm{~F}$ introducer the inner diameter of the soft-tip is effectively reduced from 0.058 inches to 0.053 inches - an $8.3 \%$ decrease in diameter. 


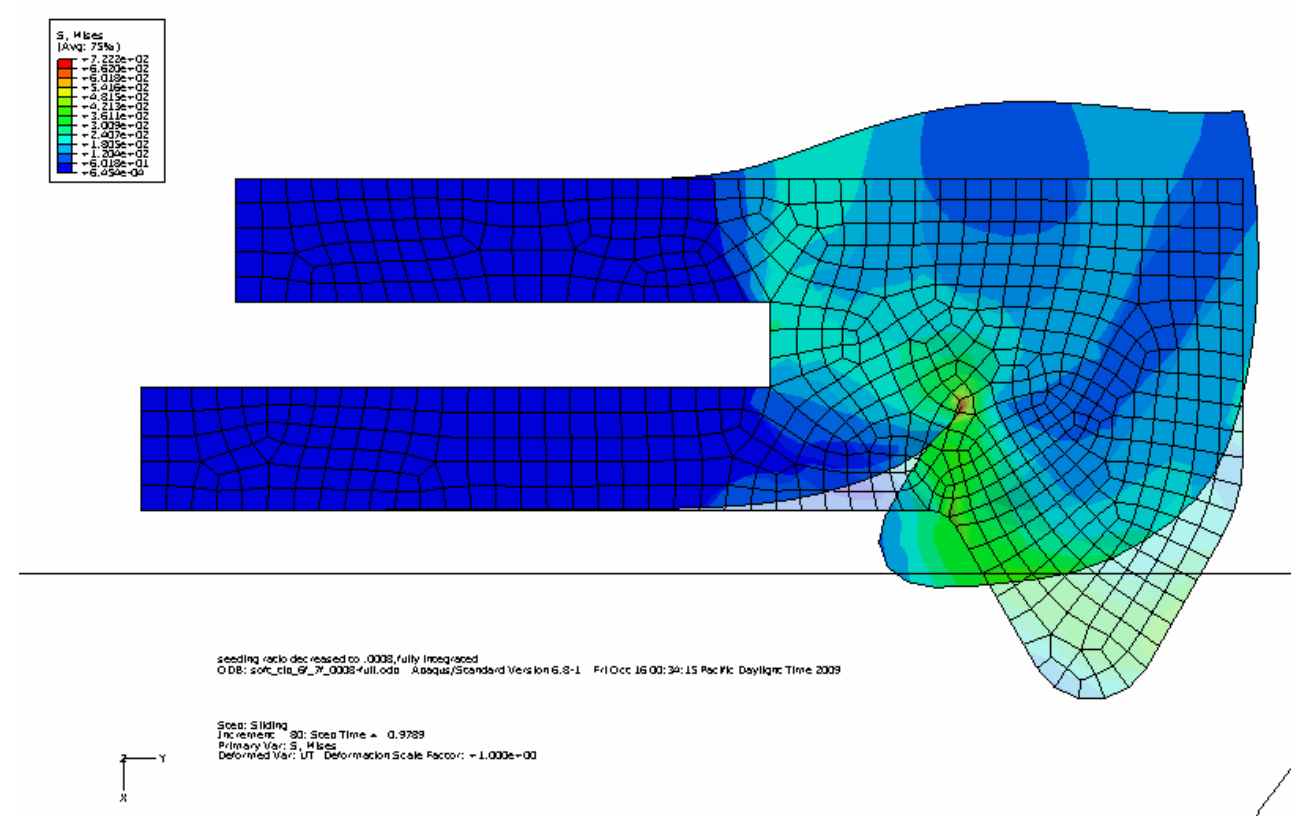

Figure 4-6. Displacement of the Advanced ICD soft-tip during insertion.

The reaction force along the Y-Axis, RF2, was recorded throughout the simulation as the $6 \mathrm{~F}$ introducer was sliding over the $6 \mathrm{~F}$ Advanced ICD soft-tip and is presented in Figure 4-7. With a seeding ratio of 0.0008 the maximum RF2 in this Advanced ICD simulation was 0.104lbf. 


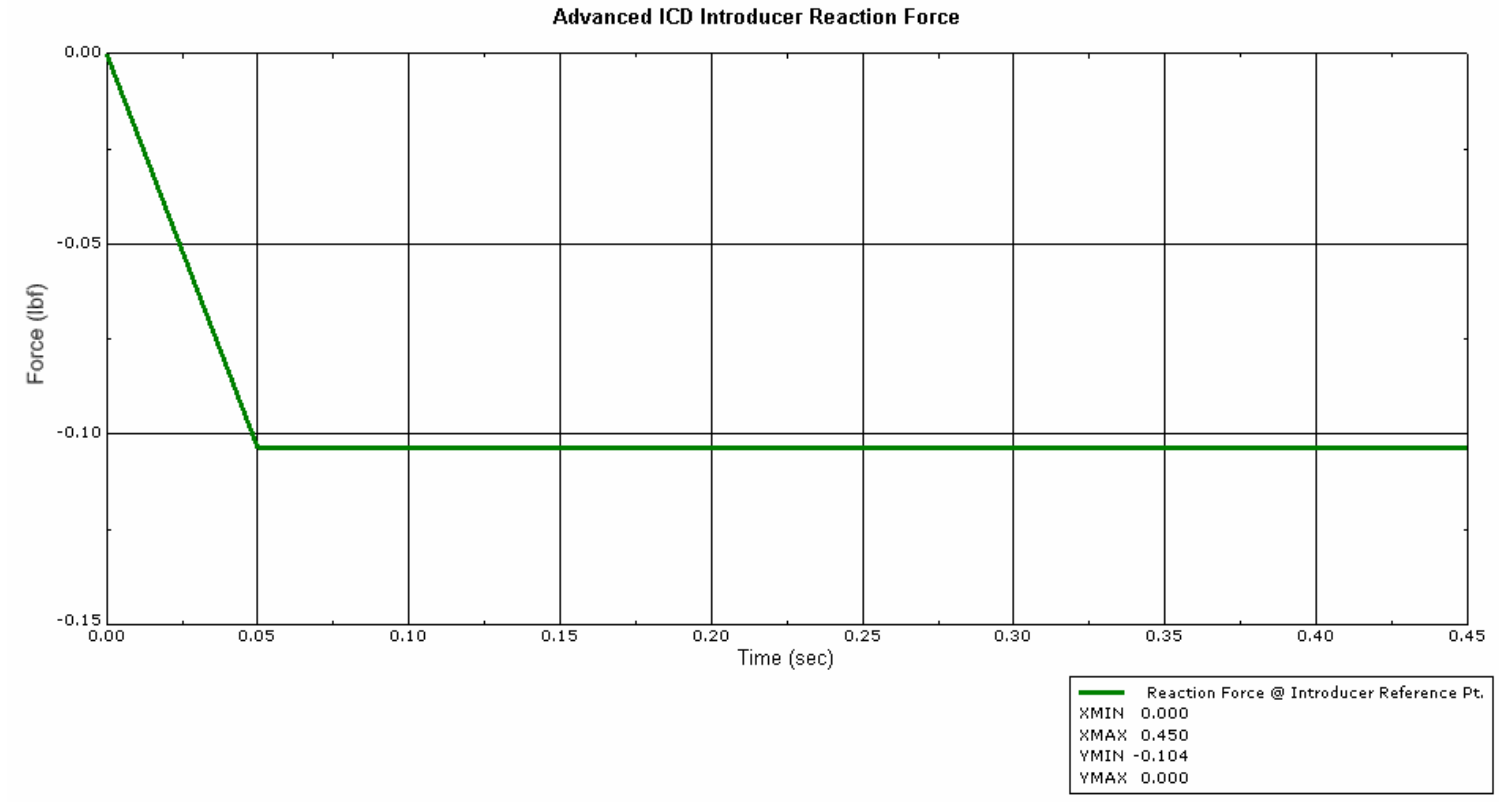

Figure 4-7. Simulated push force of the 6F Advanced ICD Soft-Tip and 6F Introducer models.

\subsection{EXPERIMENTAL RESULTS}

Every lead-type developed at St. Jude Medical is required to pass a Lead Introducer Test to verify the lead passage through a specified lead introducer size. The procedure for the lead introducer test is as follows:

1. Secure an introducer to a $1 \mathrm{lb}$ force gauge.

2. Place a stylet into the lead.

3. Grip the lead no farther than $1.0 \mathrm{in}$. from the base of the introducer.

4. With constant pressure insert the lead into the introducer - rotating the lead while inserting. Ensure that the lead does not buckle while feeding into the introducer.

5. Record the maximum force required to pass the electrode past the tip of the introducer.

The specification requires that the lead shall pass through the introducer at a load of less than $0.4 \mathrm{lb}$ with no damage to the lead or insulation. Eight (8) 7F Durata ICD leads were available for introducer testing. Due to the early stages of development, only two (2) 
6F Advanced ICD leads were available for introducer testing. The results of the lead introducer tests are shown in Tables 4-1 and 4-2.

Table 4-1. Experimental lead introducer test for 7F Durata ICD lead.

\begin{tabular}{|l|l|}
\hline Name: & Jose Lepe \\
\hline Test Date: & 2-Feb-09 \\
\hline Equipment: & Chatillon Force Gauge \\
\hline & 7F Peel-Away Introducer \\
\cline { 2 - 2 } & 7F Durata Lead \\
\cline { 2 - 2 } & Firm Stylet \\
\cline { 2 - 2 }
\end{tabular}

\begin{tabular}{|c|c|}
\hline \multicolumn{2}{|c|}{ Introducer Test } \\
\hline Serial \# & $\begin{array}{c}\text { Max. Push } \\
\text { Force (Ibs) }\end{array}$ \\
\hline AHB 12518 & 0.125 \\
\hline AHB 12518 & 0.115 \\
\hline AHB 12519 & 0.149 \\
\hline AHB 12519 & 0.132 \\
\hline AHB 12519 & 0.122 \\
\hline AHB 12506 & 0.134 \\
\hline AHB 12506 & 0.122 \\
\hline AHB 12506 & 0.120 \\
\hline avg. force & $\mathbf{0 . 1 2 7}$ \\
std. dev. & 0.011
\end{tabular}


Table 4-2. Experimental lead introducer test for 6F Advanced ICD lead.

\begin{tabular}{|l|l|}
\hline Name: & Jose Lepe \\
\hline Test Date: & 2-Feb-09 \\
\hline Equipment: & Chatillon Force Gauge \\
\cline { 2 - 2 } & 6F Peel-Away Introducer \\
\cline { 2 - 2 } & 6F Advanced ICD Lead \\
\cline { 2 - 2 } & Firm Stylet \\
\cline { 2 - 2 }
\end{tabular}

\begin{tabular}{|c|c|}
\hline \multicolumn{2}{|c|}{ Introducer Test } \\
\hline Serial \# & $\begin{array}{c}\text { Max. Push } \\
\text { Force (Ibs) }\end{array}$ \\
\hline Adv ICD 1 & 0.110 \\
\hline Adv ICD 2 & 0.121 \\
\hline & \\
\hline & \\
\hline & \\
\hline & \\
\hline & \\
\hline avg. force & $\mathbf{0 . 1 1 6}$ \\
std. dev. & 0.008
\end{tabular}

\subsection{THEORETICAL AND EXPERIMENTAL COMPARISONS}

As stated in Section 3.2 of this report, some assumptions were made when modeling the Durata and Advanced ICD Soft-Tips which may contribute to the simulation error. Overall, both models showed good correlation with the introducer test data. The maximum push force predicted in the Durata Soft-Tip simulation was $1.56 \%$ lower than the average experimental push force, but well within the standard deviation (Figure 4-8). The error resulting from the Advanced ICD Soft-Tip simulation was less accurate with a predicted maximum push force $10.34 \%$ lower than the average experimental push force for the 6F Advanced ICD lead (Figure 4-9). 


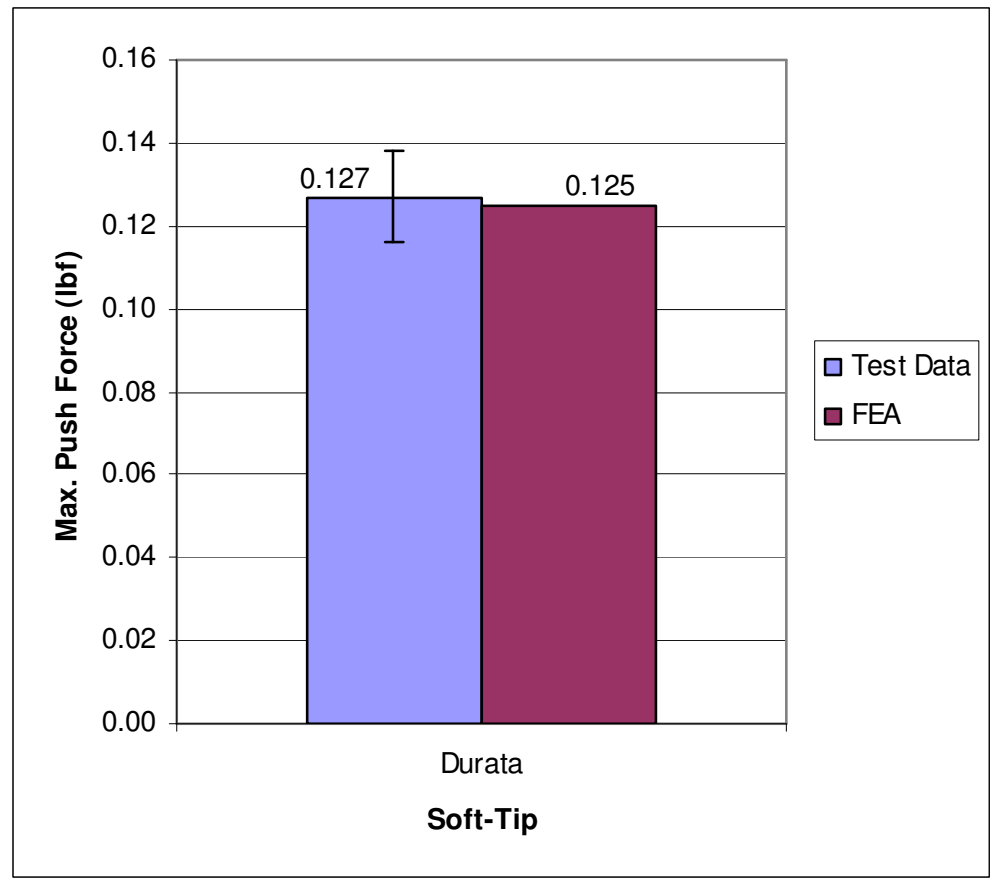

Figure 4-8. FEA and experimental maximum push force for Durata Soft-Tip.

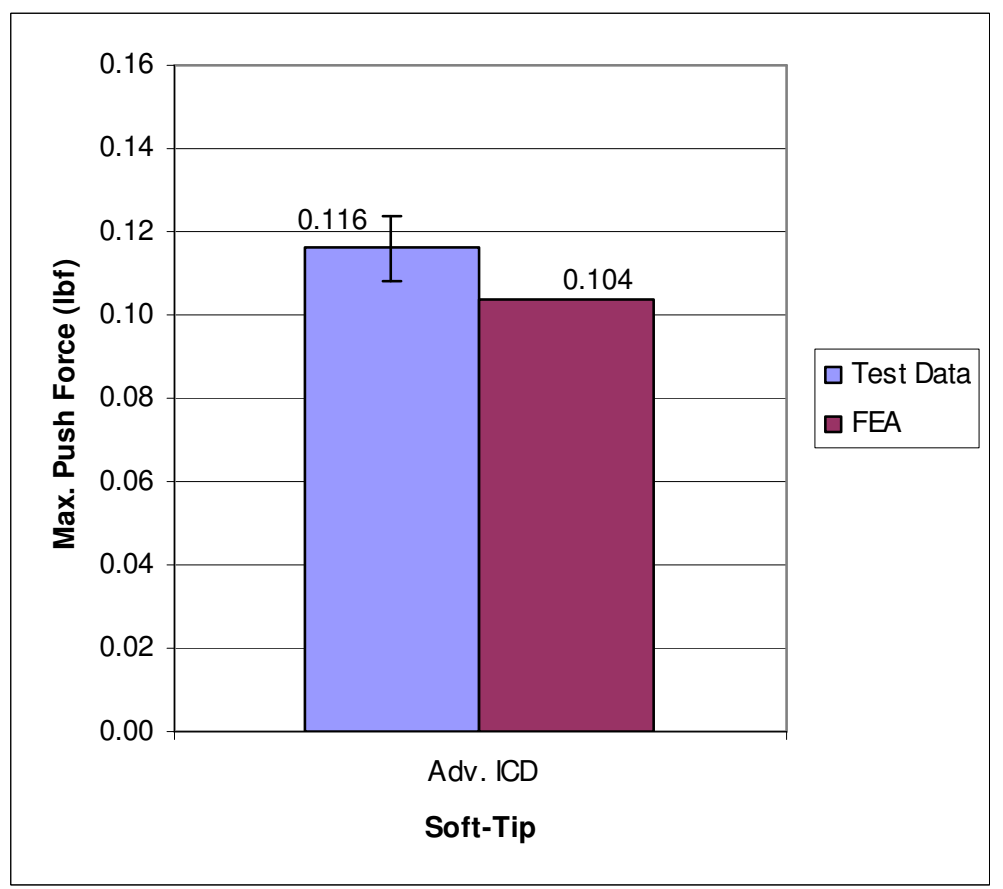

Figure 4-9. FEA and experimental maximum push force for Advanced ICD Soft-Tip. 


\section{CHAPTER 5 \\ DISCUSSION OF RESULTS}

The percent error between the physical tests and the FEA simulations are significantly different in the Durata case compared to the Advanced ICD case for a number of reasons. The distal tip subassembly of the Durata ICD lead has more symmetrical components (i.e. the marker band supporting the over-molded soft-tip is a solid ring with no cutouts), and therefore the geometry simplifications of the model had little effect on the results. The distal tip subassembly of the Advanced ICD lead on the other hand has multiple non-symmetrical cutouts in the marker band to improve delamination resistance of the insert-molded soft-tip. Results show that the simplification of the Advanced ICD marker band geometry has a direct effect on the push force. Simulation results demonstrated that the Advanced ICD model experiences increased compression of the silicone rubber as the soft-tip is passed through the introducer. The lack of shear and compression data may also contribute to the inaccuracy of the material model, and consequently, the resulting stresses.

The physical introducer test also has some variation that can contribute to the inaccuracy of the results. The introducer test is typically performed manually and can result in a variable insertion rate. Velocity is known to have an effect on the push force inserting the lead with a higher velocity will inherently result in a slightly lower push force. Slowly inserting the lead through the introducer allows static friction to take effect, thus causing the soft-tip to fold back resulting in higher compression forces between the soft-tip and the introducer. 
It is assumed that a small experimental sample size led to an inaccuracy in the experimental push force. In general, a larger sample size leads to an increase the in precision of your estimated variables. Due to the early development phase of the Advanced ICD lead, only two samples were available for testing. Any part variation inherently present between lead builds would have a significant effect on variables such as the push force. 


\section{CHAPTER 6 \\ FUTURE DIRECTIONS AND CONCLUSIONS}

\subsection{FUTURE DIRECTIONS}

Future directions of this study begin with conducting a material study that includes all silicone rubbers used in molded soft-tips, and obtaining data for all modes of deformation both in their virgin and conditioned states. By testing the material in its virgin and conditioned states, we can begin to understand how the push forces vary between a lead that has been implanted straight out of the package, and one that might have been used in engineering tests where the distal tip can experience multiple insertions through an introducer. Once all modes of deformation are obtained work can begin on defining 3D finite element soft-tip models, thus allowing further refinement in the design of a lead's distal section. In addition to developing 3D versions of the soft-tips, generating dynamic simulations would allow us to better understand how the velocity at which the lead enters the introducer affects the deformation of the soft-tip and the overall push force of the lead through the introducer.

\subsection{CONCLUSIONS}

Finite element analysis has great potential for the medical device industry. In addition to optimizing soft-tip design parameters, FEA is a versatile tool that can be used, in some cases, as a substitute for physical experimentation. It has been shown in this study, through numerical simulations and confirmed through physical testing, that FEA can be used to predict the force required to pass a lead through a specified introducer. 
Obtaining the appropriate inputs for mathematical material models is often overlooked, but has a significant impact on the accuracy and rate of convergence of your simulations - especially when simulating a hyperelastic material where several modes of deformation are needed to sufficiently define the variables in the hyperelastic material model. Although test data for only one mode of deformation was available for this study, the simulations conducted in this study were in close agreement with experimental results. Future work discussed in the previous section would undoubtedly require additional material testing. 


\section{REFERENCES}

[1] "The Conduction System." Texas Heart Institute - Heart Information Center Aug. $2009<$ http://www.texasheartinstitute.org/HIC/anatomy/conduct.cfm>.

[2] "Resources." St. Jude Medical 4 Apr. $2010<$ <ttp://www.sjm.com/resources/ resourceindex.aspx>

[3] Lindgren, Anders, and Soren Jansson. Heart Physiology and Stimulation: An Introduction. Solna, Sweden: Siemens-Elema AB, 1992.

[4] Parent, Andre. "Giovanni Aldini: From Animal Electricity to Human Brain Stimulation.” Canadian Journal of Neurological Sciences. 31 (2004): 576-584.

[5] Furman, Seymour. “The Early History of Cardiac Pacing.” Indian Pacing and Electrophysiology Journal. 2 (2002): 2-3.

[6] Kenny, Tom. The Nuts and Bolts of Cardiac Pacing. Massachusetts: Blackwell Publishing, 2005.

[7] Efimov, Igor R., Mark W. Kroll, and Patrick J. Tchou. Cardiac Bioelectric Therapy: Mechanisms and Practical Implications. New York: Springer, 2009.

[8] Ellenbogen, Kenneth. Cardiac Pacing. Massachusetts: Blackwell Science, Inc., 1996.

[9] Nelson, Randall, et al. "Leads for the ICD.” Implantable cardioverter defibrillator therapy: The engineering-clinical interface. Norwell, MA: Kluwer Academic Publishers, 1996.

[10] Von Bergen, Nicholas, et al. "'Ratchet' Syndrome, Another Etiology for Pacemaker Lead Dislodgement: A Case Report.” Heart Rhythm Society. 4 (2007): 788-789.

[11] Zielińska, Marzenna, et al. "The malposition of transvenous pacemaker lead in the left ventricle - how to avoid this rare complication." Case Report and Clinical Practice Review. 5 (2004): 358-360.

[12] Pavia, Stephen, and Bruce Wilkoff, "The management of surgical complications of pacemaker and implantable cardioverter-defibrillators." Current Opinion in Cardiology. 16 (2001): 66-71. 
[13] Nakazato, Yujt, et al. "Malposition of Screw-in Lead in the Left Ventricle." Journal of Hong Kong College of Cardiology. 8 (2000): 22-38.

[14] Seki, Hiroshi, et. al., "Malpositioning of a Pacemaker Lead to the Left Ventricle Accompanied by Posterior Mitral Leaflet Injury." Interactive Cardiovascular and Thoracic Surgery. 8 (2009): 235-237.

[15] Carlson, Mark D., Roger A. Freedman, and Paul A. Levine. "Lead Perforation: Incidence in Registries." PACE. 31 (2008): 13-15.

[16] Ellis, Christopher, and Jeffrey Rottman. "Increased Rate of Subacute Lead Complications with Small-Caliber Implantable Cardioverter-Defibrillator Leads." Heart Rhythm. 6 (2009): 619-624.

[17] "Implantable Pacing Leads and Risk of Cardiac Perforation." Guidant Corporation Product Update: 2005.

[18] Danik, Stephan, et al. "Increased Incidence of Subacute Lead Perforation Noted with One Implantable Cardioverter-Defibrillator." Heart Rhythm. 4 (2007): 439442.

[19] "Elastomer (hyperelastic) Characterization". Axel Products, Inc. 2004. $<$ http://www.axelproducts.com/pages/hyperelastic.html>

[20] Ogden, R.W. Non-Linear Elastic Deformations. New York: Dover Publications, 1984.

[21] Zhao, Yong, et al. "The Use of Non-Linear FEA Modeling to Determine the InVivo Cardiac Pacing Lead Coils for Fatigue Evaluation." 2003 Summer Bioengineering Conference. Florida: June 25-29, 2003.

[22] Dolwichai, Prapun, et al. "Hyperelastic Material Models for Finite Element Analysis with Commercial Rubber." Technology and Innovation for Sustainable Development Conference (TISD2006). Thailand: January 25-26, 2006. 


\section{BIBLIOGRAPHY}

[1] Liu, Yi, Amy E. Kerdok, and Robert D. Howe, "A Nonlinear Finite Element Model of Soft Tissue Indentation." Proceedings of Medical Simulation: International Symposium - ISMS 2004, Cambridge, MA, June 17-18, 2004, Lecture Notes in Computer Science vol. 3078, Springer-Verlag, pp67-76.

[2] Bradley, G.L., et al. "Rubber Modeling Using Uniaxial Test Data," Journal of Applied Polymer Science 81 (2001): 837-848.

[3] Altman, Peter. "Rotary bending fatigue of coils and wires used in cardiac lead design.” Journal of Biomedical Materials Research Part B: Applied Biomaterials 43 (1998): 21-37.

[4] Treloar, Leslie R. G., H. G. Hopkins, Ronald S. Rivlin, and J. M. Ball, "The Mechanics of Rubber Elasticity [and Discussions]," Proceedings of the Royal Society London A, 1976 351, 301-330.

[5] Dolwichai, Prapun and Limtragool, Jumlong. "Hyperelastic Material Models for Finite Element Analysis with Commercial Rubber." Technology and Innovation for Sustainable Development Conference (TISD2006). Thailand: January 25-26, 2006.

[6] Sivakumarin, Soori, et al. "Postpacemaker Implant Pericarditis: Incidence and Outcomes with Active-Fixation Leads." Journal of Pacing and Clinical Electrophysiology 25.5 (2002): 833-837.

[7] Trigano, Alexandre J., et al. "Incidence of Perforation and Other Mechanical Complications During Dual Active Fixation.” PACE 19.11 (1996): 1828-1831.

[8] Tang, Chuen, et al. "Initial Experience with a Co-Radial Bipolar Pacing Lead." PACE 20.7 (1997): 1800-1807.

[9] Fahy, Gerard J., et al. "Low Incidence of Lead Related Complications Associated with Nonthoracotomy Implantable Cardioverter Defibrillator Systems." PACE 18.1 (1995): 172-178.

[10] Molina, J. E., et al. "Perforation of the Right Ventricle by Transvenous Defibrillator Leads: Prevention and Treatment.” PACE 19.3 (1996): 288-292.

[11] Geyfman, Vitaly, et al. "Cardiac Tamponade as Complication of Active-Fixation Atrial Lead Perforations: Proposed Mechanism and Management Algorithm." PACE 30.4 (2007): 498-501. 
[12] Kashani, Amir, et al. "Aortic Perforation by Active-Fixation Atrial Pacing Lead." PACE 27.3 (2004): 417-418.

[13] Glikson, Michael, et al. "Clinical Surveillance of an Active Fixation, Bipolar, Polyurethane Insulated Pacing Lead, Part I: The Atrial Lead." PACE 17.9 (1994): 1499-1502.

[14] Alvarez, Vicente B., et al. "Delayed myocardial perforation following pacemaker implantation.” International Journal of Cardiology 93.1 (2004): 89-91.

[15] Velavan, P., A. Chauhan. "An unusual presentation of delayed cardiac perforation caused by atrial screw-in lead.” Heart 89.4 (2003): 364.

[16] Mond, Harry G., and David Grenz. "Implantable Transvenous Pacing Leads: The Shape of Things to Come.” PACE 27.6 (2004): 887-893.

[17] Wilkoff, Bruce L., et. al., "Pacemaker and ICD Leads: Strategies for Long-Term Management." Journal of Interventional Cardiac Electrophysiology 23.1 (2008): 59-72.

[18] Hauser, Robert G., and Adrian K. Almquist, "Learning from Our Mistakes? Testing New ICD Technology.” New England Journal of Medicine 359.24 (2008): 2517-2519.

[19] Irnich W. Stimulation electrodes. In: Schaldach M, Furman S, Hein F, Thull R (eds.), "Advances in Pacemaker Technology". Berlin, Heidelberg, SpringerValley Publishing, 1975, 247-253.

[20] Laske, Timothy G., et. al. "High Pacing Impedances: Are You Overtorquing Your Leads?” PACE 28.9 (2005): 883-891.

[21] Vlay, Stephen C. "Complications of Active-Fixation Electrodes." PACE 25.8 (2003): 1153-1154.

[22] Anderson, Sara E. "Variation in Pacing Impedance: Impact of Implant Site and Measurement Method." PACE 30.9 (2007): 1076-1082.

[23] McCotter, Craig J. "Placement of Transvenous Pacemaker and ICD Leads Across Total Chronic Occlusions," PACE 2005; 28:921-925.

[24] Grace, Lin, et al. "Severe Symptomatic Tricuspid Valve Regurgitation Due to Permanent Pacemaker or Implantable Cardioverter-Defibrillator Leads" Journal of the American College of Cardiology 45.10 (2005): 1672-1675.

[25] "The Engineer Who Could". Hopkins Medical News 7 Mar. 2010. $<$ http://www.hopkinsmedicine.org/hmn/W98/engr.html>. 
[26] Batra, R. C. "Contact of a Rubberlike Roll Cover with a Rigid Plane Surface." Proceedings-2nd International Congress on Numerical Methods for Engineering. Paris, Dec. 1980. 